\title{
Leading soft theorem for multiple gravitini
}

\author{
Diksha Jain ${ }^{a, b}$ and Arnab Rudra ${ }^{b}$ \\ ${ }^{a}$ SISSA International School for Advanced Studies and INFN - Sezione di Trieste, \\ Via Bonomea 265, Trieste 34136 Italy \\ ${ }^{b}$ International Centre for Theoretical Physics, \\ Strada Costiera 11, Trieste 34151 Italy \\ E-mail: djain@sissa.it, rudra.arnab@gmail.com
}

ABSTRACT: We compute leading soft theorem for multiple gravitini (and gravitons) in an arbitrary theory of supergravity with an arbitrary number of finite energy particles by extending Sen's approach [1,2] to fermionic symmetry. Our results are independent of the mass and the spin of the external particles. Our results are valid for any compactification of type II and Heterotic superstring theory. Our results are valid at all orders in perturbation theory for four and higher spacetime dimensions.

KeYwords: Scattering Amplitudes, Supergravity Models, Superstrings and Heterotic Strings

ARXIV EPRINT: 1811.01804 


\section{Contents}

1 Introduction $\quad 2$

1.1 Main result 3

2 Set-up 5

$\begin{array}{ll}2.1 \text { Covariant derivative } & 7\end{array}$

2.2 Vertex of one soft gravitino to matter 7

2.3 External particles 8

2.4 Coupling of two soft gravitini to a soft graviton 8

2.5 Note on Feynman diagrams 8

$\begin{array}{llr}3 & \text { Single soft gravitino } & 9\end{array}$

$\begin{array}{ll}3.1 \text { Gauge invariance } & 10\end{array}$

4 Two soft gravitini $\quad \mathbf{1 0}$

$\begin{array}{lll}4.1 & \text { Gauge invariance } & 14\end{array}$

$\begin{array}{lll}4.2 & \text { Simultaneous and consecutive soft limit } & 15\end{array}$

5 Arbitrary number of soft gravitini $\quad \mathbf{1 5}$

$\begin{array}{lll}5.1 & \text { Re-arrangement } & 17\end{array}$

$\begin{array}{lll}5.2 & \text { Gauge invariance } & 18\end{array}$

6 Two soft gravitini in the presence of central charge 19

6.1 Gauge invariance 21

6.2 Presence of soft graviton 22

$7 \quad$ Infrared divergence and soft gravitino theorem $\quad 22$

$\begin{array}{lll}7.1 & \text { Infrared divergences in } D \geq 5 & 22\end{array}$

7.2 Infrared divergences in $D=4 \quad 23$

7.2.1 Single real soft gravitino in presence of virtual graviton 23

7.2.2 Single real soft gravitino in presence of virtual graviphoton 26

$\begin{array}{ll}7.2 .3 & \text { Massless matter }\end{array}$

8 Conclusion $\quad 27$

$\begin{array}{lr}\text { A Notation and convention } & \mathbf{2 8}\end{array}$

A.1 Gamma matrix and spinor convention 28

$\begin{array}{lll}\text { A.2 } & \text { Majorana spinor } & 29\end{array}$

$\begin{array}{lr}\text { B Three soft gravitini } & 29\end{array}$

B.1 Gauge invariance 33 


\section{Introduction}

In a scattering event, a particle whose momentum in the center of mass frame is much lower than other particles is called a soft particle and the relation between the $S$-matrix with and without the soft particles is known as the soft theorem. The soft theorems capture certain universal features of the theories. Study of soft theorems is an old subject [3-13]. However, in the last few years the interest on soft theorems has been renewed because of its connection to asymptotic symmetry [14-19]. It has been argued that the flat space $S$-matrix in four dimensions should possess the symmetry of an asymptotically flat space. This symmetry is spontaneously broken and the graviton is the Goldstone boson of this spontaneously broken symmetry. Similarly, soft photon theorem can also be understood as a consequence of the large gauge transformations. These studies established the relation between seemingly different phenomena - Asymptotic symmetry, soft theorems, and memory effect. In subsequent papers the study of asymptotic symmetry was extended to higher than four dimensions $[18,20,21]$ but our understanding of asymptotic symmetries in an arbitrary dimension is far from being complete. The spacetime dimension independent treatment mostly relies on Feynman diagrammatic techniques. In this approach, one starts from a specific Lagrangian and then computes only a subclass of the Feynman diagrams which contribute to the (sub-)leading soft theorem(s). Soft photon and soft graviton theorem were computed in [22-67]. The new impetus in this direction came from Sen's work [1,2]. This method relies on covariantization of 1PI effective action with respect to the soft field. So the result does not depend on any particular Lagrangian or on asymptotic symmetry. This powerful method was used to compute sub-sub-leading soft graviton theorem [68] and also to compute multiple (sub-)leading soft graviton theorem [65]. It has been noted that the soft-photon theorem is universal at leading order $[6,7]$ and the soft-graviton theorem is universal not only in the leading order but also in the sub-leading order [69]. In a recent paper [70], the soft theorems has been investigated when two different types of massless particles are present.

In four and higher dimensions the theories of massless particles are severely constrained by Poincare invariance and Unitarity. Massless particles with spin $>2$ cannot couple minimally; they can couple only through the field strength. So the only particles which possess gauge invariance and can have minimal coupling are spin $1,3 / 2,2$. We already have a complete understanding of soft photon and soft graviton theorem. However, our knowledge of the soft gluon and the soft gravitino theorems is limited. These computations involve subtlety in the sense that the leading soft factors do not commute and their commutator is also leading order in soft momenta. At the level complexity, the soft gravitino theorem is more subtle than photon or graviton but significantly less subtle than that of the gluon. This is because even though the commutator of two soft factors is non-vanishing, the commutator of three soft factors vanishes in the case of gravitino but it does not vanish for gluon. However, for specific type of theories, soft gluon theorem can be conveniently computed using CHY formalism [71-74]. This advantage is not currently available for soft gravitino/photino. In this paper, we wish to derive the leading order soft theorem for gravitino in a general quantum field theory with local Supersymmetry in an arbitrary 
number of dimensions. Soft gravitino operator is a fermionic soft operator. Though a lot is known about bosonic soft theorems, the available literature for fermionic soft theorems is significantly little. Single soft photino theorem was computed in [75].

Amplitudes with one and two soft gravitini for four-dimensional supergravity theories were computed for a particular model in [76-78]. The result for single soft gravitino in $D=4$ can also be obtained from asymptotic symmetry [79, 80]. We generalize the result to the case with an arbitrary number of soft gravitini. In our work, we follow Sen's covariantization approach $[1,2,68]$. The advantage of this method is that it is valid for arbitrary theories, to all orders in perturbation theory and in arbitrary dimensions, as long as there is no infrared divergence. In this paper, we mostly follow the notation and conventions of [68]. We have summarized our notation and convention in section A. We find that for multiple gravitini the leading order result is universal.

An important aspect of quantum theories with massless particles is IR divergence. In $D=4$ loop diagrams suffer from IR divergences. In QED, there is a procedure to write IR finite $\mathrm{S}$ matrix element [81]. This procedure has also been understood from the perspective of asymptotic symmetries [82-84]. There has been some recent progress for quantum gravity $[85,86]$. In section 7 we discuss the IR divergence of supergravity theories. Our result is valid for any theory of supergravity in $D \geq 4 .^{1}$

Background independence of String field theory implies that String field theory in the presence of a soft field is obtained simply by deforming the world-sheet CFT by a marginal super-conformal operator which corresponds to that field. Recently Sen has proved background independence in superstring field theory [87]. So our analysis is also valid for any supersymmetric compactification of superstring theory.

\subsection{Main result}

Our main result is equation (5.1) where we have written the soft factor for arbitrary number of external soft gravitini. Consider an amplitude $\Gamma_{M+N}\left(\left\{p_{i}\right\},\left\{k_{u}\right\}\right)$ for $M$ soft gravitini and $N$ hard particles. It is related to the amplitude of $N$ hard particles $\Gamma_{N}\left(\left\{p_{i}\right\}\right)$ in the following way

$$
\Gamma_{M+N}\left(\left\{p_{i}\right\},\left\{k_{u_{i}}\right\}\right)=\left[\prod_{i=1}^{M} \mathcal{S}_{u_{i}}+\sum_{A=1}^{\lfloor M / 2\rfloor} \prod_{i=1}^{A} \mathcal{M}_{u_{i} v_{i}} \prod_{j=1}^{M-2 A} \mathcal{S}_{r_{j}}\right] \Gamma_{N}\left(\left\{p_{i}\right\}\right)+\mathcal{O}\left(1 / k^{M-1}\right)
$$

Various terms in this expression are explained below

1. $p_{i}$ are the momenta of the hard particles, $k_{u}$ are momenta of the soft particles

2. $\mathcal{S}_{u}$ is the soft factor for single soft gravitino. It is given by

$$
\mathcal{S}_{u}=\kappa \sum_{i=1}^{N}\left(\frac{\epsilon_{\mu}^{(u) \alpha} p_{i}^{\mu}}{p_{i} \cdot k_{u}} \mathcal{Q}_{\alpha}\right)
$$

Here $\kappa$ is the gravitational coupling constant. $\epsilon_{\mu}^{(u) \alpha}$ is the polarization of the $u^{\text {th }}$ gravitino; it has a Lorentz vector index \& a majorana spinor index and it is grassmann

\footnotetext{
${ }^{1}$ In $D \leq 3$ there is no graviton and gravitino.
} 
odd. The gravitino polarization (in the harmonic gauge) satisfies the transversality condition and gamma traceless condition.

$$
\left(k_{u}\right)^{\mu} \epsilon_{\mu}^{(u) \alpha}=0 \quad, \quad \gamma_{\alpha \beta}^{\mu} \epsilon_{\mu}^{(u) \beta}=0
$$

$\mathcal{Q}_{\alpha}$ are the supersymmetry charges/generators. The single soft gravitino factor in four dimensional theories was also computed in [77, 79, 80]. Since $\mathcal{S}_{u}$ is a product of two grassmann odd quantities, it is grassmann even. Two single soft factors do not commute with each other.

$$
\mathcal{S}_{u} \mathcal{S}_{v} \neq \mathcal{S}_{v} \mathcal{S}_{u}
$$

3. Whenever there is more than one gravitino, they can combine pairwise to give a soft graviton which in-turn couples to the hard particles. $\mathcal{M}_{u v}$ encodes these type of contributions. The explicit expression for $\mathcal{M}_{u v}$ is given by

$$
\mathcal{M}_{u v}=\kappa^{2} \sum_{i=1}^{N} \frac{1}{2} \frac{\epsilon_{\mu}^{(u)} p_{i} \epsilon_{\nu}^{(v)}}{p_{i} \cdot\left(k_{u}+k_{v}\right)}\left[\frac{p_{i}^{\mu} p_{i}^{\nu}}{p_{i} \cdot k_{v}}+\frac{1}{2} \frac{\eta^{\mu \nu} p_{i} \cdot\left(k_{u}-k_{v}\right)}{k_{u} \cdot k_{v}}+\frac{\left(k_{v}^{\mu} p_{i}^{\nu}-k_{u}^{\nu} p_{i}^{\mu}\right)}{k_{u} \cdot k_{v}}\right]
$$

$\mathcal{M}_{u v}$ is neither symmetric nor anti-symmetric in its (particle-)indices

$$
\mathcal{M}_{u v} \neq \pm \mathcal{M}_{v u}
$$

4. Since the single soft factors for gravitino do not commute, the final expression for the arbitrary number of soft gravitini depends on the choice of ordering of external soft gravitini. In section 5.1, we demonstrate that any order can be obtained from any other ordering. However, our expression is not manifestly symmetric in various soft gravitini.

5. The first term is the product of single-soft gravitino factors. The single-soft factors appear in a particular order and the explicit form of second piece changes depending on the ordering of soft factors because two soft factors do not commute.

6. In the second term, $\lfloor M / 2\rfloor$ denotes the greatest integer which is less than or equal to $M / 2$. A counts the number of pairs of gravitini giving a soft graviton. The subscripts $\left\{r_{j}, u_{i}, v_{i}\right\}$ can take values from $1, \ldots, M$ and $v_{i}>u_{i}$ and $r_{j}$ 's are also ordered with the largest $r_{j}$ appearing on the right.

7. The supersymmetry algebra may contain central charges. In this case, the gravitino super-multiplet contains graviphoton. In the presence of central charge, there are additional contributions to $\mathcal{M}_{u v}$ due to graviphoton couplings. In the presence of central charge the expression of $\mathcal{M}_{u v}$ is modified as follows

$$
\begin{aligned}
\mathcal{M}_{u v} \longrightarrow \widetilde{\mathcal{M}}_{u v}=\mathcal{M}_{u v}+ & \frac{\kappa^{2}}{2} \sum_{i=1}^{N} e_{i} \frac{\epsilon_{\mu}^{(u)} \mathcal{Z} \epsilon_{\nu}^{(v)}}{p_{i} \cdot\left(k_{u}+k_{v}\right)} \\
& \times\left[\frac{p_{i}^{\mu} p_{i}^{\nu}}{p_{i} \cdot k_{v}}+\frac{1}{2} \frac{\eta^{\mu \nu} p_{i} \cdot\left(k_{u}-k_{v}\right)}{k_{u} \cdot k_{v}}+\frac{\left(k_{v}^{\mu} p_{i}^{\nu}-k_{u}^{\nu} p_{i}^{\mu}\right)}{k_{u} \cdot k_{v}}\right]
\end{aligned}
$$


$e_{i}$ is the charge of the $i^{\text {th }}$ external state under symmetry generated by graviphoton. $\mathcal{Z}$ is an element of the Clifford algebra such that $\mathcal{Z}_{\alpha}{ }^{\beta}$ commutes with all other element of the Clifford algebra and ${ }^{2}$

$$
\mathcal{Z}_{\alpha \beta}=\mathcal{Z}_{\beta \alpha}
$$

We checked the gauge invariance of (1.1).

In the presence of soft graviton, we have to multiply the above expression by soft factors of the graviton. For $M_{1}$ soft gravitini and $M_{2}$ soft gravitons equation (1.1) is modified as given below

$$
\Gamma_{N+M_{1}+M_{2}}\left(\left\{p_{i}\right\},\left\{k_{r}\right\}\right)=\left[\prod_{j=1}^{M_{2}} \widetilde{\mathcal{S}}_{u_{j}}\right]\left[\prod_{i=1}^{M_{1}} \mathcal{S}_{u_{i}}+\sum_{A=1}^{\left\lfloor M_{1} / 2\right\rfloor} \prod_{i=1}^{A} \mathcal{M}_{u_{i} v_{i}} \prod_{j=1}^{M_{1}-2 A} \mathcal{S}_{r_{j}}\right] \Gamma_{N}\left(\left\{p_{i}\right\}\right)
$$

$\widetilde{\mathcal{S}}_{u}$ is the leading soft factor for graviton. It given by

$$
\widetilde{\mathcal{S}}_{u}=\kappa \sum_{i=1}^{N}\left(\frac{\zeta_{\mu \nu}^{(u)} p_{i}^{\mu} p_{i}^{\nu}}{p_{i} \cdot k_{u}}\right)
$$

here $\zeta_{\mu \nu}$ is the polarization of soft graviton.

The rest of the paper is organized as follows. In section 2, we derive the vertices from the 1PI effective action. Then we start with the simplest case of single soft gravitino in section 3. We show the gauge invariance of the expression. Then we compute the expression for the two soft gravitini in section 4. The coupling of the gravitini to the graviton is essential to show the gauge invariance of the expression for two soft gravitini. Then we write down the expression for multiple soft gravitini in section 5. We do not present any derivation of this result. We check the gauge invariance of this expression. Our conjectured result is based on the computation in section 3, section 4 and appendix B. In section 6 , we derive the contribution to the soft theorem, when the supersymmetry algebra contains central charges. In section 7 we discuss infrared divergence in supergravity and we show that the soft gravitino theorem is not affected by the IR divergence. Then we present our brief conclusion and potential future directions.

\section{Set-up}

We are interested in deriving the leading order soft theorem for gravitino in an arbitrary theory of supergravity. We follow the approach of Sen $[1,2]$. As in $[1,2]$ we treat finite energy gravitino and soft gravitino differently. One can always do that at the level of tree amplitudes. So, we consider 1PI effective action and replace all the derivatives by covariant derivative with respect to the soft gravitino.

Our starting point is a globally super-symmetric 1PI effective action which is invariant under some number of Majorana supersymmetries. ${ }^{3}$ So the usual (dimension-dependent)

\footnotetext{
${ }^{2}$ Spinor indices are raised and lowered using charge-conjugation matrix.

${ }^{3}$ From Coleman-Mandula theorem and HLS theorem, the maximum number of super-charge is 32 .
} 
restriction for the existence of a globally super-symmetric action applies. We promote the global supersymmetry to a local one by replacing all the derivatives with covariant derivatives. At the leading order, only the minimal coupling of gravitino with matter fields contribute. We do not assume anything about the multiplet in which matter fields are sitting. Our analysis is valid for the matter in any supersymmetry multiplet.

Let $\Phi_{m}$ be any quantum field which transforms under some reducible representation of the Poincare group, supersymmetry, and the internal symmetry group(s). The transform of the fields under the global supersymmetry is given by

$$
\Phi_{m} \longrightarrow \Phi_{m}+\mathbf{i}\left(\theta^{\alpha} \mathcal{Q}_{\alpha}\right)_{m}{ }^{n} \Phi_{n}
$$

$\mathcal{Q}_{\alpha}$ are supersymmetry generators. They satisfy the following algebra

$$
\left\{\mathcal{Q}_{\alpha}, \mathcal{Q}_{\beta}\right\}=-\frac{1}{2} \gamma_{\alpha \beta}^{\mu} P_{\mu}
$$

Here $P_{\mu}$ is the momentum generator. The indices $\alpha, \beta$ are the collection of all possible spinor indices, not the indices for the minimal spinor (of that dimension). So, in a theory with more than one supersymmetry, $\mathcal{Q}_{\alpha}$ are the collection of all the super-charges. Gamma matrices are in Majorana representation and symmetric in the spinor indices.

Now we will evaluate the vertex that describes the coupling of a soft gravitino to any hard particle. We start from the quadratic term of the 1PI effective action [2]

$$
S=\frac{1}{2} \int \frac{d^{d} p_{1}}{(2 \pi)^{D}} \frac{d^{d} p_{2}}{(2 \pi)^{D}} \Phi_{m}\left(p_{1}\right) \mathcal{K}^{m n}\left(p_{2}\right) \Phi_{n}\left(p_{2}\right)(2 \pi)^{D} \delta^{(D)}\left(p_{1}+p_{2}\right)
$$

The kinetic term is invariant under global supersymmetry transformation. This implies

$$
\mathcal{K}^{m_{1} m_{3}}\left(\mathcal{Q}_{\alpha}\right)_{m_{3}}{ }^{m_{2}}+\mathcal{K}^{m_{3} m_{2}}\left(\mathcal{Q}_{\alpha}\right)_{m_{3}}{ }^{m_{1}}=0
$$

Later we will need the propagator. Let's assume it has the following form:

$$
\Xi(q)\left(q^{2}+M^{2}\right)^{-1}
$$

where $\Xi(q)$ is defined as

$$
\Xi(q)=\mathbf{i}\left(q^{2}+M^{2}\right) \mathcal{K}^{-1}(q)
$$

and $M$ is some arbitrary mass parameter. ${ }^{4}$ From (2.4) we get,

$$
\Xi^{m_{1} m_{3}}\left(\mathcal{Q}_{\alpha}\right)_{m_{3}}^{m_{2}}+\Xi^{m_{3} m_{2}}\left(\mathcal{Q}_{\alpha}\right)_{m_{3}}^{m_{1}}=0
$$

We write down two more relations which will be useful later

$$
\begin{aligned}
\mathcal{K}^{m_{1} m_{2}}(-p) \Xi_{m_{2} m_{3}}(-p) & =\mathbf{i}\left(p^{2}+M^{2}\right) \delta^{m_{1}} m_{3} \\
\frac{\partial \mathcal{K}^{m_{1} m_{2}}(-p)}{\partial p_{\mu}} \Xi_{m_{2} m_{3}}(-p) & =-\mathcal{K}^{m_{1} m_{2}}(-p) \frac{\partial \Xi_{m_{2} m_{3}}(-p)}{\partial p_{\mu}}+2 \mathbf{i} p^{\mu} \delta^{m_{1}}{ }_{m_{3}}
\end{aligned}
$$

\footnotetext{
${ }^{4}$ We have already used $M$ for number of soft-particles. Since the mass-parameter does not appear extensively, we also $M$ for mass-parameter.
} 


\subsection{Covariant derivative}

In super-gravity theories, the super-covariant derivative [88] is given by

$$
\mathcal{D}_{a}=E_{a}{ }^{\mu}\left(\partial_{\mu}-\mathbf{i} \Psi_{\mu}{ }^{\alpha} \mathcal{Q}_{\alpha}-\mathbf{i} \frac{1}{2} \omega_{\mu}{ }^{c d} \mathcal{J}_{c d}\right)
$$

The local-supersymmetry transformation of the vielbein $e_{\mu}^{a}$ and the gravitino $\Psi_{\mu \alpha}$ are given by

$$
\begin{aligned}
\delta e_{\mu}^{a} & =\frac{1}{2} \theta \gamma^{a} \Psi_{\mu} \\
\delta \Psi_{\mu \alpha} & =\mathcal{D}_{\mu} \theta_{\alpha}=\partial_{\mu} \theta_{\alpha}+\frac{1}{4} \omega_{\mu a b} \gamma^{a b} \theta_{\alpha}
\end{aligned}
$$

Here $\theta$ is the local supersymmetry parameter. We consider the covariant derivative with respect to the soft fields only. So we consider a small fluctuation with soft momenta [2]

$$
\begin{aligned}
E_{a}{ }^{\mu} & =\delta_{a}{ }^{\mu}-\kappa \zeta_{a}{ }^{\mu} e^{\mathrm{i} k \cdot x} \\
\Psi_{\mu}{ }^{\alpha} & =\kappa \epsilon_{\mu}{ }^{\alpha} e^{\mathrm{i} k \cdot x}
\end{aligned}
$$

Here $\kappa$ is the gravitational coupling constant. At linear order in fluctuation of the soft fields we get the following expression for the super-covariant derivative

$$
\mathcal{D}_{a}=\partial_{a}-\kappa \zeta_{a}{ }^{\mu} \partial_{\mu}-\mathbf{i} \kappa \epsilon_{a}^{\alpha} \mathcal{Q}_{\alpha}-\mathbf{i} \kappa \frac{1}{2} \omega_{a}{ }^{c d}\left(\zeta_{a}{ }^{\mu}\right) \mathcal{J}_{c d}
$$

\subsection{Vertex of one soft gravitino to matter}

The coupling of one soft gravitino to matter fields at linear order can be found by covariantizing the derivative in (2.3). Due to the interaction with gravitino, the momenta of hard particle changes by $\delta q=-\kappa \epsilon_{\mu}^{\alpha} \mathcal{Q}_{\alpha}$. So the quadratic part of the 1PI effective action changes as follows (2.3) [2]:

- $\delta^{(D)}\left(p_{1}+p_{2}\right)$ gets replaced by $\delta^{(D)}\left(p_{1}+p_{2}+k\right)$ where $k$ is the momenta of soft gravitino.

- The change in kinetic operator $\mathcal{K}^{m n}$ due to shift in momenta has to be substituted.

So we get

$$
S^{(L)}=\frac{1}{2} \int \frac{d^{d} p_{1}}{(2 \pi)^{D}} \frac{d^{d} p_{2}}{(2 \pi)^{D}} \Phi_{m}\left(p_{1}\right)\left[-\frac{\partial \mathcal{K}\left(p_{2}\right)}{\partial p_{2 \mu}} \kappa \epsilon_{\mu}^{\alpha} \mathcal{Q}_{\alpha}\right]^{m n} \Phi_{n}\left(p_{2}\right)(2 \pi)^{D} \delta^{(D)}\left(p_{1}+p_{2}+k\right)
$$

So the vertex is given by

$$
-\left[\mathbf{i} \kappa \frac{\partial \mathcal{K}\left(p_{i}\right)}{\partial p_{i \mu}} \epsilon_{\mu}^{\alpha} \mathcal{Q}_{\alpha}\right]^{m n}
$$




\subsection{External particles}

Since we compute only $S$-matrix elements, all the external particles satisfy on-shell and transversality condition. The external particle of polarization $\varepsilon_{i, m}$ and momenta $p_{i}$ satisfies the conditions:

$$
\begin{aligned}
\varepsilon_{i, m} \mathcal{K}^{m n}(q) & =0 \\
p_{i}^{2}+M_{i}^{2} & =0
\end{aligned}
$$

\subsection{Coupling of two soft gravitini to a soft graviton}

When we have more than one soft gravitino, we need to consider the minimal coupling of gravitino with graviton. To derive this vertex, we followed [2]. The graviton coupling to any matter field can be written as:

$$
\begin{aligned}
S= & \frac{1}{2} \int \frac{d^{D} k_{1}}{(2 \pi)^{D}} \frac{d^{D} k_{2}}{(2 \pi)^{D}}(2 \pi)^{D} \delta^{(D)}\left(k_{1}+k_{2}+p\right) \\
& \times \Phi_{m}\left(k_{1}\right)\left[-\zeta_{\mu \nu} k_{2}^{\nu} \frac{\partial}{\partial k_{2 \mu}} \mathcal{K}^{m n}\left(k_{2}\right)+\frac{1}{2}\left(p_{b} \zeta_{a \mu}-p_{a} \zeta_{b \mu}\right) \frac{\partial}{\partial k_{2 \mu}} \mathcal{K}^{m p}\left(k_{2}\right)\left(\mathcal{J}^{a b}\right)_{p}{ }^{n}\right] \Phi_{n}\left(k_{2}\right)
\end{aligned}
$$

where $\zeta_{\mu \nu}$ is the graviton polarization.

The kinetic term for the gravitino, in the harmonic gauge, is given by

$$
\mathcal{K}^{\mu \alpha ; \nu \beta}(p)=\left(p_{\rho} \gamma^{\rho}\right)^{\alpha \beta} \eta^{\mu \nu}
$$

The angular momentum generator is

$$
\left(\mathcal{J}^{a b}\right)_{\mu, \alpha}{ }^{\nu, \beta}=\left(\mathcal{J}_{V}^{a b}\right)_{\mu}{ }^{\nu} \delta_{\alpha}{ }^{\beta}+\left(\mathcal{J}_{S}^{a b}\right)_{\alpha}{ }^{\beta} \delta_{\mu}{ }^{\nu}
$$

where $\mathcal{J}_{V}^{a b}$ and $\mathcal{J}_{S}^{a b}$ are angular momentum generator in vector and spinor representations respectively.

$$
\begin{array}{ll}
\left(\mathcal{J}_{V}^{a b}\right)_{\mu}{ }^{\nu}=\delta^{a}{ }_{\mu} \eta^{b \nu}-\delta^{b}{ }_{\mu} \eta^{a \nu} & \\
\left(\mathcal{J}_{S}^{a b}\right)_{\alpha}{ }^{\beta}=-\frac{1}{2}\left(\gamma^{a b}\right)_{\alpha}{ }^{\beta} & \gamma^{a b} \equiv \frac{1}{2}\left(\gamma^{a} \gamma^{b}-\gamma^{b} \gamma^{a}\right)
\end{array}
$$

Our gamma matrix convention is given in (A.2). Our convention is that all the particles are incoming; the gravitino has momentum $k_{1}$ and $k_{2}$ and the graviton has momenta $p$. The momentum conservation implies

$$
p+k_{1}+k_{2}=0
$$

So the vertex $\left(\mathcal{V}^{\mu \nu ; \mu_{1} \mu_{2}}\right)^{\alpha \beta}$ is given by

$$
-\mathbf{i} \kappa\left[k_{2}^{\mu_{2}}\left(\gamma^{\mu_{1}}\right)^{\alpha \beta} \eta^{\mu \nu}+\frac{1}{4}\left(p_{d} \delta_{c}^{\mu_{2}}-p_{c} \delta_{d}^{\mu_{2}}\right)\left(\gamma^{\mu_{1}} \gamma^{c d}\right)^{\alpha \beta} \eta^{\mu \nu}+\left(p^{\mu} \eta^{\nu \mu_{2}}-p^{\nu} \eta^{\mu \mu_{2}}\right)\left(\gamma^{\mu_{1}}\right)^{\alpha \beta}\right]
$$

\subsection{Note on Feynman diagrams}

We use a red double-arrowed line for soft-gravitino, a blue wavy line to denote soft gravitons, a violet wavy line for graviphoton, Cyan double arrowed ${ }^{5}$ line for hard fermionic particles (including hard gravitini) and black line to denote hard bosonic particles.

\footnotetext{
${ }^{5}$ We use a double arrowed line for Majorana particles because they are their own anti-particle; they only have $\mathbb{Z}_{2}$ charge.
} 


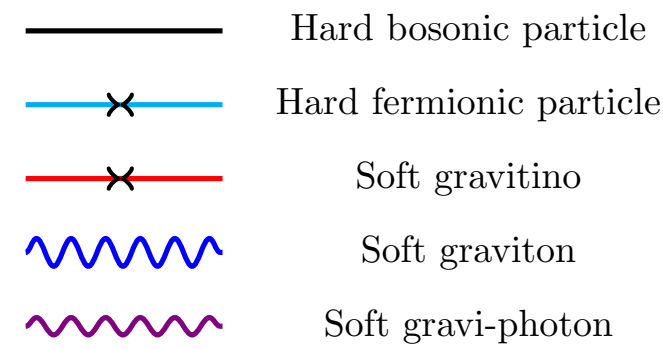

Figure 1. Conventions for Feynman diagrams.

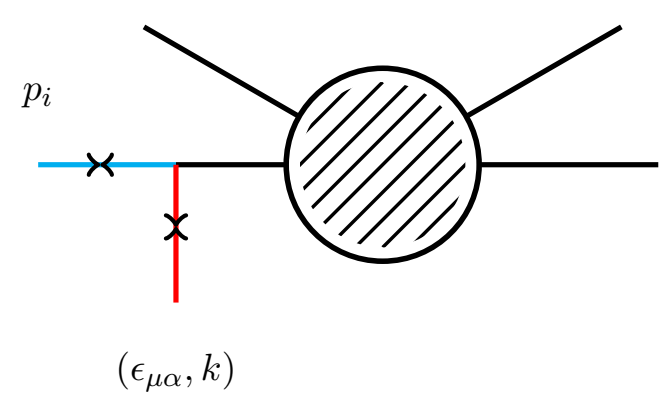

Figure 2. Feynman diagram for single soft gravitino.

\section{Single soft gravitino}

In this section, we compute the leading order contribution to soft gravitino theorem due to one soft gravitino. This result for $D=4$ was first derived in [77] and was reproduced from the analysis of asymptotic symmetries in $[79,80]$. The only diagram that contributes to this process is depicted in figure 2.

The expression for the propagator is given in equation (2.5). In this diagram, the propagator carries momenta $p_{i}+k$ and $M_{i}$ is the mass of the $i$-th particle. Let us denote the corresponding propagator by $\Xi_{m_{i} n_{i}}\left(p_{i}+k\right)$. The contribution to figure 2 is given by:

$$
\begin{aligned}
& \Gamma_{N+1}^{m_{1} \ldots m_{N}}\left(\left\{p_{i}\right\}, k\right) \\
& \quad=\left[\mathbf{i} \kappa \sum_{i=1}^{N}\left(\frac{\partial \mathcal{K}\left(-p_{i}\right)}{\partial p_{i \mu}} \epsilon_{\mu}^{\alpha} \mathcal{Q}_{\alpha}\right)^{m_{i} n_{i}} \frac{\Xi_{n_{i} \tilde{n}_{i}}}{\left(p_{i}+k\right)^{2}+M_{i}^{2}}\right] \Gamma_{N}^{m_{1} \ldots m_{i-1} \tilde{n}_{i} m_{i+1} \ldots m_{N}}\left(\left\{p_{i}\right\}\right) \\
& \quad=\left[\mathbf{i} \kappa \sum_{i=1}^{N}\left(\frac{\partial \mathcal{K}\left(-p_{i}\right)}{\partial p_{i \mu}} \epsilon_{\mu}^{\alpha} \mathcal{Q}_{\alpha}\right)^{m_{i} n_{i}} \frac{\Xi_{n_{i} \tilde{n}_{i}}}{\left(2 p_{i} \cdot k\right)}\right] \Gamma_{N}^{m_{1} \ldots m_{i-1} \tilde{n}_{i} m_{i+1} \ldots m_{N}}\left(\left\{p_{i}\right\}\right)
\end{aligned}
$$

where in the second step, we have used the on-shell condition (2.15b) for external hard particle and the fact that gravitino is soft. Now we will use (2.7) and (2.8) to simplify the expression

$$
\begin{aligned}
\left(\frac{\partial \mathcal{K}\left(-p_{i}\right)}{\partial p_{i \mu}} \epsilon_{\mu}^{\alpha} \mathcal{Q}_{\alpha}\right)^{m_{i} n_{i}} \Xi_{n_{i} \tilde{n}_{i}} & =\epsilon_{\mu}^{\alpha}\left(\frac{\partial \mathcal{K}\left(-p_{i}\right)}{\partial p_{i \mu}} \mathcal{Q}_{\alpha} \Xi\right)^{m_{i}} \tilde{n}_{i}=-\epsilon_{\mu}^{\alpha}\left(\frac{\partial \mathcal{K}\left(-p_{i}\right)}{\partial p_{i \mu}} \Xi \mathcal{Q}_{\alpha}\right)^{m_{i}} \tilde{n}_{i} \\
& =-\epsilon_{\mu}^{\alpha}\left(-\mathcal{K}\left(-p_{i}\right) \frac{\partial \Xi}{\partial p_{i \mu}} \mathcal{Q}_{\alpha}+2 \mathbf{i} p_{i}^{\mu} \mathcal{Q}_{\alpha}\right)^{m_{i}} \tilde{n}_{i}
\end{aligned}
$$


From first step to second step we have used (2.7) and from second step to third step we have used (2.8). Now the first term drops out because of the on-shell condition (2.15a). Hence we obtain [77]

$$
\Gamma_{N+1}^{m_{1} \ldots m_{N}}\left(\left\{p_{i}\right\}, k\right)=\left[\kappa \sum_{i=1}^{N}\left(\frac{p_{i}^{\mu} \epsilon_{\mu}^{\alpha}}{p_{i} \cdot k} \mathcal{Q}_{\alpha}\right)^{m_{i}} \tilde{n}_{i}\right] \Gamma_{N}^{m_{1} \ldots m_{i-1} \tilde{n}_{i} m_{i+1} \ldots m_{N}}\left(\left\{p_{i}\right\}\right)
$$

Soft operator. We define the soft operator $\mathcal{S}_{u}[77]$ as

$$
\mathcal{S}_{u}=\kappa \sum_{i=1}^{N}\left(\frac{p_{i}^{\mu} \epsilon_{\mu}^{(u) \alpha}}{p_{i} \cdot k_{u}} \mathcal{Q}_{\alpha}\right)
$$

where $u$ labels the soft gravitino. So the above result can be re-written as:

$$
\Gamma_{N+1}^{m_{1} \ldots m_{N}}\left(\left\{p_{i}\right\}, k\right)=\left[\mathcal{S}^{m_{i}} \tilde{n}_{i}\right] \Gamma_{N}^{m_{1} \ldots m_{i-1} \tilde{n}_{i} m_{i+1} \ldots m_{N}}\left(\left\{p_{i}\right\}\right)
$$

\subsection{Gauge invariance}

As a consistency check, we check the gauge invariance of equation (3.3). We put pure gauge polarization for the gravitino

$$
\epsilon_{\alpha \mu}=k_{\mu} \theta_{\alpha}
$$

Here $\theta_{\alpha}$ is a Majorana spinor. For pure gauge gravitino the amplitude should vanish. From (3.3), we obtain

$$
\theta^{\alpha} \sum_{i=1}^{N}\left(\mathcal{Q}_{\alpha}\right)^{m_{i}} \tilde{n}_{i} \Gamma_{N}^{m_{1} \ldots m_{i-1} \tilde{n}_{i} m_{i+1} \ldots m_{N}}\left(p_{i}\right)=0
$$

This is the ward-identity for the global super-symmetry.

\section{Two soft gravitini}

Now we will consider the amplitude with $N$ hard particles and 2 soft gravitini. There are essentially four different types of Feynman diagrams which contribute to this process

1. The class of diagrams where the two soft gravitini are attached to different external legs (for example, figure 3). These diagrams are easy to evaluate. The computation for these type of diagrams is essentially the same as single soft gravitino. The contribution from figure 3 is given by

$$
\kappa^{2} \sum_{i=1}^{N} \frac{\epsilon_{\mu}^{(1) ; \alpha} p_{i}^{\mu}}{p_{i} \cdot k_{1}} \mathcal{Q}_{\alpha} \sum_{j=1 ; j \neq i}^{N} \frac{\epsilon_{\nu}^{(2) ; \beta} p_{i}^{\nu}}{p_{j} \cdot k_{2}} \mathcal{Q}_{\beta} \Gamma\left(\left\{p_{i}\right\}\right)
$$

2. The class of diagrams where both of the soft gravitini are attached to the same external leg. There are mainly three types of such diagrams - (figure 4, figure 5, figure 6). Figure 4, figure 5 denote the diagrams where the soft gravitino directly attaches the same hard-particles. These two diagrams differ only in order of attaching to the hard particle. Figure 6 captures the process when the soft gravitini combine to give a soft graviton and then the soft graviton attaches to the hard particles.

Now will evaluate these diagrams. 


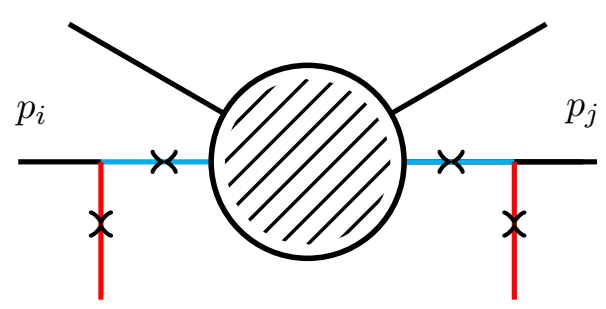

$$
\left(\epsilon_{\mu \alpha}^{(1)}, k_{1}\right) \quad\left(\epsilon_{\nu \beta}^{(2)}, k_{2}\right)
$$

Figure 3. Feynman diagram for double soft gravitini - I.

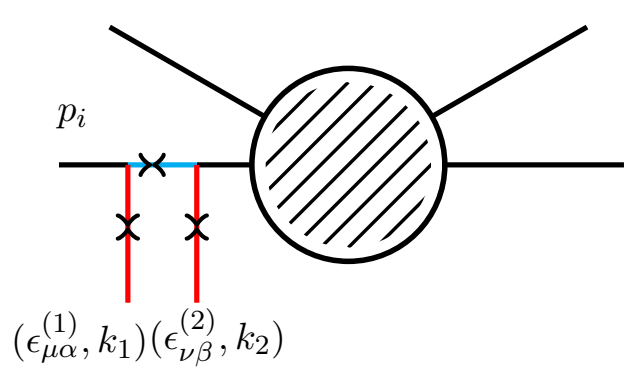

Figure 4. Feynman diagram for double soft gravitini - II.

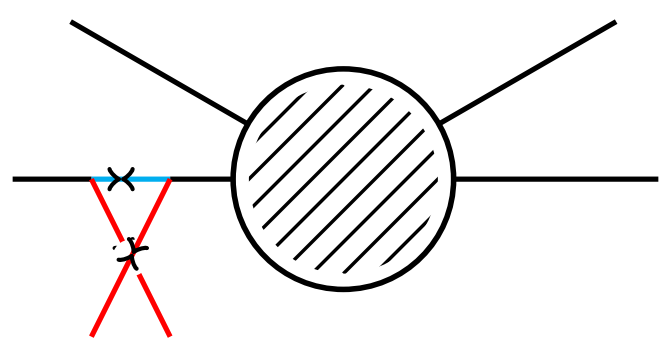

Figure 5. Feynman diagram for double soft gravitini — III.

Evaluation of figure 4 . The contribution from the Feynman diagram in figure 4 is given by

$$
\begin{aligned}
\Gamma_{N+2}^{(1)}=\kappa^{2} \sum_{i=1}^{N} \frac{\partial \mathcal{K}^{m p}\left(-p_{i}\right)}{\partial p_{i \mu}} & \frac{\left[\epsilon_{\mu}^{(1) ; \alpha} \mathcal{Q}_{\alpha} \Xi\left(-p_{i}-k_{1}\right)\right]_{p q}}{\left(2 p_{i} \cdot k_{1}\right)} \\
& \times \frac{\partial \mathcal{K}^{q r}\left(-p_{i}-k_{1}\right)}{\partial p_{i \nu}} \frac{\left[\epsilon_{\nu}^{(2) ; \beta} \mathcal{Q}_{\beta} \Xi\left(-p_{i}-k_{1}-k_{2}\right)\right]_{r s}}{\left(2 p_{i} \cdot\left(k_{1}+k_{2}\right)\right)} \Gamma_{N}\left(\left\{p_{i}\right\}\right)
\end{aligned}
$$

Using (2.7) and (2.8) we can simplify this expression and we get

$$
\kappa^{2} \sum_{i=1}^{N} \frac{\epsilon_{\mu}^{(1) ; \alpha} p_{i}^{\mu}}{p_{i} \cdot k_{1}} \frac{\epsilon_{\nu}^{(2) ; \beta} p_{i}^{\nu}}{p_{i} \cdot\left(k_{1}+k_{2}\right)} \mathcal{Q}_{\alpha} \mathcal{Q}_{\beta} \Gamma_{N}\left(\left\{p_{i}\right\}\right)
$$




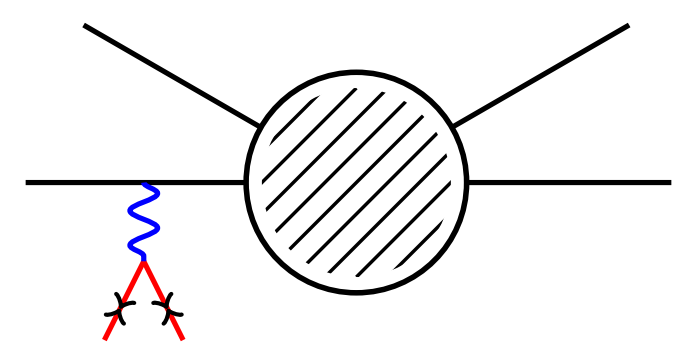

Figure 6. Feynman diagram for double soft gravitini - IV.

Evaluation of figure 5. The contribution due to figure 5 can obtained from equation (4.2) by interchanging $1 \longleftrightarrow 2$

$$
\begin{aligned}
\Gamma_{N+2}^{(2)}\left(\left\{p_{i}\right\}, k_{1}, k_{2}\right) & =\kappa^{2} \sum_{i=1}^{N} \frac{\epsilon_{\nu}^{(2) ; \beta} p_{i}^{\nu}}{p_{i} \cdot k_{2}} \frac{\epsilon_{\mu}^{(1) ; \alpha} p_{i}^{\mu}}{p_{i} \cdot\left(k_{1}+k_{2}\right)} \mathcal{Q}_{\beta} \mathcal{Q}_{\alpha} \Gamma_{N}\left(\left\{p_{i}\right\}\right) \\
& =\kappa^{2} \sum_{i=1}^{N} \frac{\epsilon_{\mu}^{(1) ; \alpha} p_{i}^{\mu}}{p_{i} \cdot k_{2}} \frac{\epsilon_{\nu}^{(2) ; \beta} p_{i}^{\nu}}{p_{i} \cdot\left(k_{1}+k_{2}\right)}\left[\mathcal{Q}_{\alpha} \mathcal{Q}_{\beta}+\frac{1}{2}\left(\not p_{i}\right)_{\alpha \beta}\right] \Gamma_{N}\left(\left\{p_{i}\right\}\right)
\end{aligned}
$$

Evaluation of figure 6. Now we would like to evaluate the figure 6. This diagram denotes the process when two soft gravitini interact first to produce a soft graviton which then attaches to any of the external legs. The contributions from these kinds of processes are given by

$$
\begin{aligned}
\Gamma_{N+2}^{(3)}\left(\left\{p_{i}\right\}, k_{1}, k_{2}\right)= & -\left[\epsilon_{\mu}^{(1) \alpha}\left(\mathcal{V}^{\mu \nu ; \mu_{1} \mu_{2}}\right)_{\alpha \beta} \epsilon_{\nu}^{(2) \beta}\right] \\
& \times\left[\left(\frac{\mathbf{i}}{2}\right) \frac{\eta_{\mu_{1} \nu_{1}} \eta_{\mu_{2} \nu_{2}}+\eta_{\mu_{1} \nu_{2}} \eta_{\mu_{2} \nu_{1}}-\frac{2}{D-2} \eta_{\mu_{1} \mu_{2}} \eta_{\nu_{1} \nu_{2}}}{2\left(k_{1} \cdot k_{2}\right)}\right] \\
& \times\left[-\mathbf{i} \kappa p_{i}^{\nu_{1}} \frac{\partial \mathcal{K}}{\partial p_{i \nu_{2}}} \frac{\Xi}{2 p_{i} \cdot\left(k_{1}+k_{2}\right)}\right] \Gamma_{N}\left(\left\{p_{i}\right\}\right)
\end{aligned}
$$

where the first square bracket denotes gravitino-gravitino-graviton vertex, the second one is the graviton propagator and the third one is the matter- soft graviton -matter vertex. Using the explicit expression for $\left(\mathcal{V}^{\mu \nu ; \mu_{1} \mu_{2}}\right)_{\alpha \beta}$ from (2.21) and simplifying the above expression, we get

$$
\begin{aligned}
\Gamma_{N+2}^{(3)} & \left(\left\{p_{i}\right\}, k_{1}, k_{2}\right) \\
= & \frac{\kappa^{2}}{2} \sum_{i=1}^{N} \epsilon_{\mu}^{(1) \alpha}\left(\not p_{i}\right)_{\alpha \beta}\left[-\eta^{\mu \nu} p_{i} \cdot k_{2}-\frac{1}{2} \eta^{\mu \nu}\left(k_{1}+k_{2}\right)_{d} p_{i e} \gamma^{d e}+\left(k_{2}^{\mu} p_{i}^{\nu}-k_{1}^{\nu} p_{i}^{\mu}\right)\right] \epsilon_{\nu}^{(2) \beta} \\
& \times\left[\frac{1}{\left(p_{i} \cdot\left(k_{2}+k_{1}\right)\right)\left(k_{1} \cdot k_{2}\right)}\right] \Gamma_{N}\left(\left\{p_{i}\right\}\right)
\end{aligned}
$$

After simplifying the second term and using gamma-traceless condition for gravitino, we get

$$
\Gamma_{N+2}^{(3)}=\kappa^{2}\left[\sum_{i=1}^{N} \mathcal{C}_{12}\left(p_{i}\right) \frac{1}{\left(p_{i} \cdot\left(k_{2}+k_{1}\right)\right)}\right] \Gamma_{N}\left(\left\{p_{i}\right\}\right)
$$


here we have introduced $\mathcal{C}_{12}$ where $\mathcal{C}_{u v}\left(p_{i}\right)$ is defined as follows

$$
\mathcal{C}_{u v}\left(p_{i}\right)=\frac{1}{2} \epsilon_{\mu}^{(u)} p_{i} \epsilon_{\nu}^{(v)}\left[\frac{1}{2} \frac{\eta^{\mu \nu} p_{i} \cdot\left(k_{u}-k_{v}\right)}{k_{u} \cdot k_{v}}+\frac{\left(k_{v}^{\mu} p_{i}^{\nu}-k_{u}^{\nu} p_{i}^{\mu}\right)}{k_{u} \cdot k_{v}}\right]
$$

From the property of the gamma matrices it follows that $\mathcal{C}_{u v}\left(p_{i}\right)$ is symmetric in its particle indices

$$
\epsilon_{\mu}^{(u)} p_{i} \epsilon_{\nu}^{(v)}=-\epsilon_{\mu}^{(v)} p_{i} \epsilon_{\nu}^{(u)} \Longrightarrow \mathcal{C}_{u v}\left(p_{i}\right)=\mathcal{C}_{v u}\left(p_{i}\right)
$$

Total contribution. Now we add the contributions from (4.1), (4.3), (4.4) and (4.7) to get the full answer for two soft gravitini. The total contribution can be written as

$$
\Gamma_{N+2}\left(\left\{p_{i}\right\}, k_{1}, k_{2}\right)=\left[\mathcal{S}_{1} \mathcal{S}_{2}+\mathcal{M}_{12}\right] \Gamma_{N}\left(\left\{p_{i}\right\}\right)
$$

we have already defined $\mathcal{S}_{u}$ in (3.4). $\mathcal{M}_{u v}$ is defined as follows

$$
\mathcal{M}_{u v}=\kappa^{2} \sum_{i=1}^{N} \frac{1}{2} \frac{\epsilon_{\mu}^{(u)} p_{i} \epsilon_{\nu}^{(v)}}{p_{i} \cdot\left(k_{u}+k_{v}\right)}\left[\frac{p_{i}^{\mu} p_{i}^{\nu}}{p_{i} \cdot k_{v}}+\frac{1}{2} \frac{\eta^{\mu \nu} p_{i} \cdot\left(k_{u}-k_{v}\right)}{k_{u} \cdot k_{v}}+\frac{\left(k_{v}^{\mu} p_{i}^{\nu}-k_{u}^{\nu} p_{i}^{\mu}\right)}{k_{u} \cdot k_{v}}\right]
$$

\section{Some properties of $\mathcal{S}_{u}$ and $\mathcal{M}_{u v}$.}

- Two soft operators do not commute

$$
\left[\mathcal{S}_{u}, \mathcal{S}_{v}\right]=-\frac{\kappa^{2}}{2} \sum_{i=1}^{N}\left[\left(\epsilon_{\mu}^{(u) ; \alpha} \not p_{i ; \alpha \beta} \epsilon_{\nu}^{(v) ; \beta}\right) \frac{p_{i}^{\mu}}{p_{i} \cdot k_{u}} \frac{p_{i}^{\nu}}{p_{i} \cdot k_{v}}\right]
$$

- While writing the result for two soft gravitini, we could have chosen the other ordering of soft factors but both results should match i.e.

$$
\mathcal{S}_{u} \mathcal{S}_{v}+\mathcal{M}_{u v}=\mathcal{S}_{v} \mathcal{S}_{u}+\mathcal{M}_{v u}
$$

Above equation can be explicitly verified by noting that:

$$
\mathcal{M}_{v u}-\mathcal{M}_{u v}=\kappa^{2} \sum_{i=1}^{N} \frac{\epsilon^{(u)} \cdot p_{i}}{p_{i} \cdot k_{u}} \frac{\epsilon^{(v)} \cdot p_{i}}{p_{i} \cdot k_{v}}\left(-\frac{1}{2} \not p_{i}\right)
$$

We already computed $\mathcal{S}_{u} \mathcal{S}_{v}-\mathcal{S}_{v} \mathcal{S}_{u}$ in (4.12). Hence (4.13) is satisfied.

- Three soft operators satisfy Jacobi identity.

$$
\left[\mathcal{S}_{u},\left[\mathcal{S}_{v}, \mathcal{S}_{w}\right]\right]+\left[\mathcal{S}_{v},\left[\mathcal{S}_{w}, \mathcal{S}_{u}\right]\right]+\left[\mathcal{S}_{w},\left[\mathcal{S}_{u}, \mathcal{S}_{v}\right]\right]=0
$$

In this particular case, each term in the above equation is individually zero.

$$
\left[\mathcal{S}_{u},\left[\mathcal{S}_{v}, \mathcal{S}_{w}\right]\right]=0
$$

This is not true for soft gluon operator(s). Though (4.15) is true for soft gluon operator, (4.16) does not hold for soft gluon operator. This fact makes the computation of the soft factors for multiple soft gluon even more cumbersome.

- Some more properties of $\mathcal{M}_{u v}$ are listed below

$$
\begin{aligned}
\mathcal{M}_{u v} & \neq \pm \mathcal{M}_{v u} \\
\mathcal{M}_{u_{1} v_{1}} \mathcal{M}_{u_{2} v_{2}} & =\mathcal{M}_{u_{2} v_{2}} \mathcal{M}_{u_{1} v_{1}} \\
\mathcal{S}_{w} \mathcal{M}_{u v} & =\mathcal{M}_{u v} \mathcal{S}_{w}
\end{aligned}
$$




\subsection{Gauge invariance}

As a consistency check, we check the gauge invariance of the result obtained in (4.10). The right-hand side should vanish when one puts any of the gravitini as a pure gauge. Here we will put $\epsilon^{(2)}$ as a pure gauge and check if r.h.s. vanishes or not.

$$
\epsilon_{\mu}^{(2) \alpha}=k_{2 \mu} \theta_{2}^{\alpha}
$$

So for pure gauge, the first term in (4.10) vanishes because $\mathcal{Q}_{\beta}$ directly hits $\Gamma_{N}\left(\left\{p_{i}\right\}\right)$ and gives zero due to supersymmetry ward-identity (3.7). The second piece gives:

$$
\begin{aligned}
& \mathcal{M}_{12}\left(\epsilon_{1}^{\mu \alpha}, k_{2}^{\mu} \theta_{2}^{\alpha}\right) \\
& =\kappa^{2} \sum_{i=1}^{N} \frac{1}{2} \frac{\epsilon_{\mu}^{(1)} p_{i} \theta^{(2)}}{p_{i} \cdot\left(k_{1}+k_{2}\right)}\left[\frac{p_{i}^{\mu} p_{i} \cdot k_{2}}{p_{i} \cdot k_{2}}+\frac{1}{2} \frac{k_{2}^{\mu} p_{i} \cdot\left(k_{1}-k_{2}\right)}{k_{1} \cdot k_{2}}+\frac{\left(k_{2}^{\mu}\left(k_{2} \cdot p_{i}\right)-k_{2} \cdot k_{1} p_{i}^{\mu}\right)}{k_{1} \cdot k_{2}}\right] \\
& =\kappa^{2} \sum_{i=1}^{N} \frac{1}{2} \epsilon_{\mu}^{(1)} p_{i} \theta^{(2)}\left[\frac{1}{2} \frac{k_{2}^{\mu}}{k_{1} \cdot k_{2}}\right]=0
\end{aligned}
$$

where in the last step we have used momentum conservation $\sum_{i=1}^{N} p_{i}=0$.

One should be able to show the gauge invariance when $\epsilon^{(1)}$ is pure gauge. But in this case, first term in (4.10) does not give ward-identity directly and also $\mathcal{M}_{12}$ term does not vanish. But one can check that the sum is gauge invariant. Alternative we can use (4.13) to express the amplitude in the other ordering of soft factors

$$
\Gamma_{N+2}\left(\left\{p_{i}\right\}, k_{1}, k_{2}\right)=\left[\mathcal{S}_{2} \mathcal{S}_{1}+\mathcal{M}_{21}\right] \Gamma_{N}\left(\left\{p_{i}\right\}\right)
$$

In this representation, it is obvious that the r.h.s. vanishes for pure-gauge $\epsilon^{(1)}$. In general,

$$
\begin{aligned}
& \mathcal{M}_{u v}\left(\epsilon_{u}^{\mu \alpha}, k_{v}^{\mu} \theta_{v}^{\alpha}\right)=0 \\
& \mathcal{M}_{u v}\left(k_{u}^{\mu} \theta_{u}^{\alpha}, \epsilon_{v}^{\mu \alpha}\right) \neq 0
\end{aligned}
$$

To express the result for an arbitrary number of soft gravitini, we always choose an ordering amongst the external gravitini. The gauge invariance would be manifest when one puts the last gravitino as pure gauge. Using relations of the form (4.13), one can check the gauge invariance for pure gauge configuration of any soft particle.

At this point, we would like to emphasize that the combined contribution from figure 4 and 5 is not gauge-invariant. Only after adding the contribution from figure 6 the answer becomes gauge invariant. A different way to state the same result is that the existence of massless spin $3 / 2$ particles which interact with other fields at low momenta requires an interacting massless spin 2 particle at low energy. This point was first elucidated in [77].

Symmetrized form the amplitude. The expression for the soft factor in (4.10) is not manifestly symmetric on the gravitini. That form was useful to prove gauge invariance. Now we use (4.15) and (4.16) to write the answer in a form which is manifestly symmetric 
on the gravitini

$$
\begin{aligned}
& \Gamma_{N+2}\left(\left\{p_{i}\right\}, k_{1}, k_{2}\right) \\
& =\frac{1}{2}\left[\mathcal{S}_{1} \mathcal{S}_{2}+\mathcal{S}_{2} \mathcal{S}_{1}+\mathcal{M}_{12}+\mathcal{M}_{21}\right] \Gamma_{N}\left(\left\{p_{i}\right\}\right) \\
& =\left[\frac{1}{2}\left(\mathcal{S}_{1} \mathcal{S}_{2}+\mathcal{S}_{2} \mathcal{S}_{1}\right)\right. \\
& \left.\quad \quad+\kappa^{2} \sum_{i=1}^{N} \frac{1}{p_{i} \cdot\left(k_{1}+k_{2}\right)}\left[\mathcal{C}_{12}\left(p_{i}\right)+\frac{1}{4}\left(p_{i} \cdot \epsilon^{(1)}\right) p_{i}\left(\epsilon^{(2)} \cdot p_{i}\right) \frac{p_{i} \cdot\left(k_{1}-k_{2}\right)}{\left(p_{i} \cdot k_{2}\right)\left(p_{i} \cdot k_{1}\right)}\right]\right] \Gamma_{N}\left(\left\{p_{i}\right\}\right)
\end{aligned}
$$

Apart from the last term, other terms are clearly symmetric under the exchange $1 \longleftrightarrow 2$.

\subsection{Simultaneous and consecutive soft limit}

When there are more than one soft particles, there are various ways in which one can take the soft limit. ${ }^{6}$ Consider the amplitude with $N$ hard particles with momenta $\left\{p_{i}\right\}$ and two soft particles with momenta $k_{1}$ and $k_{2}\left(\Gamma_{N+2}\left(\left\{p_{i}\right\}, k_{1}, k_{2}\right)\right)$. Then the consecutive soft limit is defined as the limit in which the momenta are taken to be soft one after another. So for two soft particles, this can be done in two different ways

$$
\lim _{k_{1} \rightarrow 0} \lim _{k_{2} \rightarrow 0} \Gamma_{N+2}\left(\left\{p_{i}\right\}, k_{1}, k_{2}\right) \quad, \quad \lim _{k_{2} \rightarrow 0} \lim _{k_{1} \rightarrow 0} \Gamma_{N+2}\left(\left\{p_{i}\right\}, k_{1}, k_{2}\right)
$$

Alternatively, one can take simultaneous limit where one takes both $k_{1}$ and $k_{2}$ to zero keeping $k_{1} / k_{2}$ fixed

$$
\lim _{k_{1}, k_{2} \rightarrow 0} \Gamma_{N+2}\left(\left\{p_{i}\right\}, k_{1}, k_{2}\right)
$$

In this paper we have focused on the simultaneous limit. If the single soft factors mutually commute (i.e. if the generators of the gauge symmetry commute) then the simultaneous limit is the same as the consecutive limit. For example, in the case of photon these two limits give the same answer. However, if the symmetry generators do not commute then these two limits differ. In our case, the supersymmetry generators do not commute. For example, if we take the consecutive limit by taking $k_{1}$ to be soft first then the Feynman diagram in figure 5 does not contribute because the soft particle (with momentum $k_{1}$ ) in figure 5 is being emitted from an internal line. Hence the total contribution in this case is different from the case when we take simultaneous soft limit.

\section{$5 \quad$ Arbitrary number of soft gravitini}

Now we consider the amplitude with an arbitrary number of soft gravitini. In this case, the following type of diagrams can contribute:

- When some soft gravitini attach on one external leg and some on another external $\operatorname{leg}(\mathrm{s})$, but none of them form pairs to give soft graviton as shown in figure 7 .

\footnotetext{
${ }^{6}$ We are thankful to the unknown referee for pointing out this issue.
} 


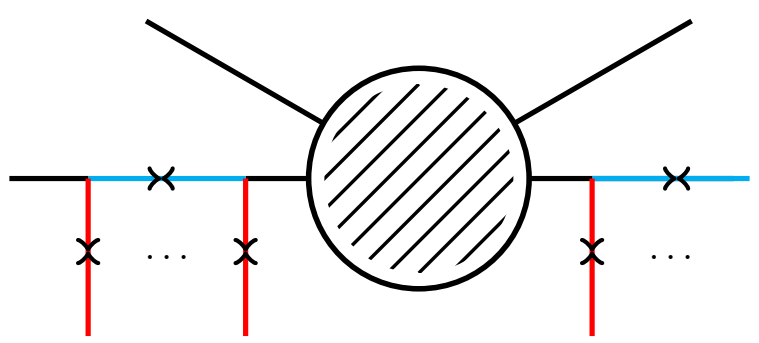

Figure 7. Feynman diagram for multiple soft gravitini - I.

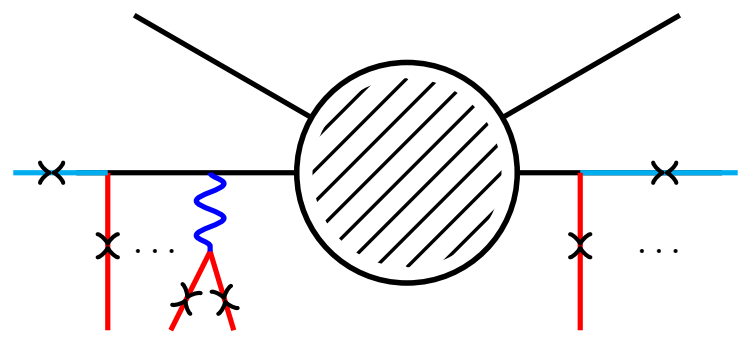

Figure 8. Feynman diagram for multiple soft gravitini - II.

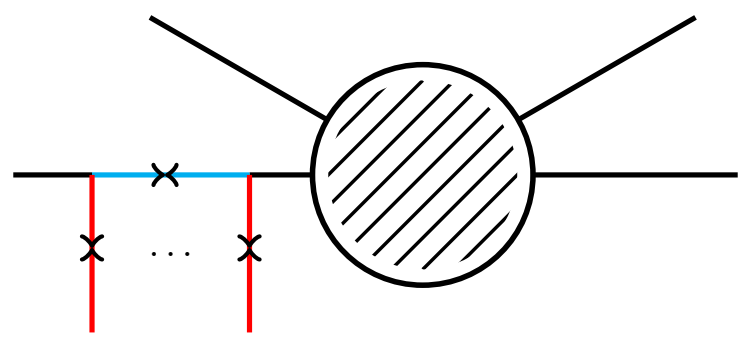

Figure 9. Feynman diagram for multiple soft gravitini - III.

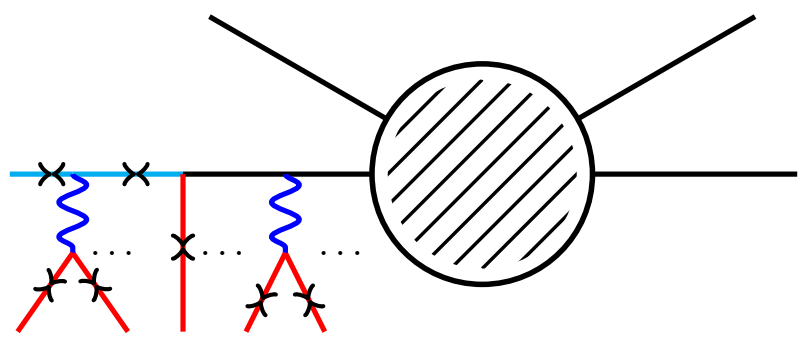

Figure 10. Feynman diagram for multiple soft gravitini - IV.

- When some soft gravitini attach on one external leg and some on another external $\operatorname{leg}(\mathrm{s})$ and some form pairs to give soft graviton as shown in figure 8.

- All gravitini attach on the same external leg, but none of them form pairs to give soft graviton as shown in figure 9 .

- Some gravitini form pairs and give a soft graviton while some attach directly to external leg as shown in figure 10.

We performed explicit computation for three soft gravitini, which is shown in appendix B. By looking at the pattern followed in two and three soft gravitini case, we 
propose the following expression for $M$-soft gravitini.

$$
\Gamma_{N+M}\left(\left\{p_{i}\right\},\left\{k_{u_{i}}\right\}\right)=\left[\prod_{i=1}^{M} \mathcal{S}_{u_{i}}+\sum_{A=1}^{\lfloor M / 2\rfloor} \prod_{i=1}^{A} \mathcal{M}_{u_{i} v_{i}} \prod_{j=1}^{M-2 A} \mathcal{S}_{r_{j}}\right] \Gamma_{N}\left(\left\{p_{i}\right\}\right)
$$

where $\lfloor M / 2\rfloor$ denotes the greatest integer which is less than or equal to $M / 2$. Now we will explain various terms.

1. The first term is very similar to the leading soft factor for multiple soft photons or multiple soft gravitons. The other terms are there because of the fact that soft gravitino factors do not commute. We always write the first factor in a particular order, for example, $\mathcal{S}_{u_{1}}, \ldots, \mathcal{S}_{u_{M}} u_{1}<u_{2} \ldots<u_{M}$ and then the particular form of the second term depends on this choice of ordering for the first term. This way to write in particular ordering also turns out to be convenient to check gauge invariance.

2. In the second term, $A$ counts the number of pairs of gravitini giving a soft graviton. For each pair, we have a factor of $\mathcal{C}_{u v}$ coming from gravitino-graviton-gravitino vertex which combines with a factor due to the use of anti-commutation relation to bring the first term in particular order, to give $\mathcal{M}_{u v}$. The subscripts $\left\{r_{j}, u_{i}, v_{i}\right\}$ can take values from $1, \ldots, M$ and $v_{i}>u_{i}$ and $r_{j}$ 's are also ordered with the largest $r_{j}$ appearing on the right.

The disadvantage of the expression (5.1) is that it depends on the ordering of the external soft gravitini. The expression is not manifestly invariant under alternation of the ordering. Now, we will show that the expression is actually invariant under rearrangement. We can go to any particular ordering starting from any other ordering. Our strategy is as follows:

1. We first show that any two consecutive entries can be interchanged.

2. By repeating this operation (of interchanging any two consecutive entries) many times, we can obtain any ordering starting from any other ordering. ${ }^{7}$

\subsection{Re-arrangement}

Here we show that any two consecutive terms of equation (5.1) can be interchanged. Consider the $i^{\text {th }}$ and $(i+1)^{\text {th }}$ particle. We write the expression (5.1)

$$
\begin{aligned}
\Gamma_{N+M}\left(\left\{p_{i}\right\}\right. & \left.,\left\{k_{u}\right\}\right)=\left[\mathcal{S}_{u_{1}} \ldots \mathcal{S}_{u_{i}} \mathcal{S}_{u_{i+1}} \ldots \mathcal{S}_{u_{M}}+\mathcal{M}_{u_{1} u_{2}} \mathcal{S}_{u_{3}} \ldots \mathcal{S}_{u_{i}} \mathcal{S}_{u_{i+1}} \ldots \mathcal{S}_{u_{M}}\right. \\
& +\mathcal{M}_{u_{2} u_{3}} \mathcal{S}_{u_{1}} \ldots \mathcal{S}_{u_{i}} \mathcal{S}_{u_{i+1}} \ldots \mathcal{S}_{u_{M-1}} \mathcal{S}_{u_{M}}+\ldots+\mathcal{M}_{u_{1} u_{i}} \mathcal{S}_{u_{2}} \ldots \mathcal{S}_{u_{i+1}} \ldots \mathcal{S}_{u_{M-1}} \mathcal{S}_{u_{M}} \\
& +\mathcal{M}_{u_{2} u_{i}} \mathcal{S}_{u_{1}} \ldots \mathcal{S}_{u_{i+1}} \ldots \mathcal{S}_{u_{M-1}} \mathcal{S}_{u_{M}}+\ldots+\mathcal{M}_{u_{i} u_{i+1}} \mathcal{S}_{u_{1}} \ldots \ldots \mathcal{S}_{u_{M-1}} \mathcal{S}_{u_{M}} \\
& \left.+\mathcal{M}_{u_{1} u_{2}} \mathcal{M}_{u_{3} u_{4}} . \mathcal{S}_{u_{i}} \mathcal{S}_{u_{i+1}} \ldots \mathcal{S}_{u_{M-1}} \mathcal{S}_{u_{M}}+. .+\mathcal{M}_{u_{1} u_{2}} \ldots \mathcal{M}_{u_{i} u_{i+1}} \ldots \mathcal{M}_{u_{M-1} u_{M}}\right] \\
& \times \Gamma_{N}\left(\left\{p_{i}\right\}\right)
\end{aligned}
$$

Here the $i^{\text {th }}$ and $(i+1)^{\text {th }}$ particle can appear only in three different ways

\footnotetext{
${ }^{7}$ Theorem 2.1 in this note gives a proof of the above statement.
} 
- Possibility $I$ : both the $i^{\text {th }}$ and $(i+1)^{\text {th }}$ gravitini appear as $\mathcal{S}$ factor

$$
\left[A \mathcal{S}_{u_{i}} \mathcal{S}_{u_{i+1}} B\right] \Gamma_{N}\left(\left\{p_{i}\right\}\right)
$$

where $A$ and $B$ involves all the other $M-2$ gravitini. The other gravitions appear as ordered multiplications of $\mathcal{S}_{u}$ and $\mathcal{M}_{v w}$ 's in all possible ways.

- Possibility II: both the $i^{\text {th }}$ and $(i+1)^{\text {th }}$ gravitino appear in $\mathcal{M}_{u v}$ together

$$
\left[\widetilde{A} \mathcal{M}_{u_{i} u_{i+1}} \widetilde{B}\right] \Gamma_{N}\left(\left\{p_{i}\right\}\right)
$$

Here $\tilde{A}$ and $\tilde{B}$ involves all the other $M-2$ gravitini. Again the other gravitions appear as ordered multiplications of $\mathcal{S}_{u}$ and $\mathcal{M}_{v w}$ 's in all possible ways. This would imply

$$
A=\widetilde{A} \quad, \quad B=\widetilde{B}
$$

So same $A$ and $B$ appear in (5.3) and in (5.4). Adding (5.3) and (5.4) we get

$$
\left[A\left(\mathcal{S}_{u_{i}} \mathcal{S}_{u_{i+1}}+\mathcal{M}_{u_{i} u_{i+1}}\right) B\right] \Gamma_{N}\left(\left\{p_{i}\right\}\right)
$$

- Possibility III: at least one of them appears as $\mathcal{M}$ and if both of them appear in $\mathcal{M}_{u v}$, they do not appear together. The possibility of both of them to appear together in $\mathcal{M}_{u v}$ has already been taken into account in possibility II.

$$
\sum_{j=1, j \neq i, i+1}^{N}\left[\mathcal{M}_{u_{j} u_{i}} C_{i+1}\left(\epsilon_{u_{i+1}}\right)+\mathcal{M}_{u_{j} u_{i+1}} C_{i}\left(\epsilon_{u_{i}}\right)\right] \Gamma_{N}\left(\left\{p_{i}\right\}\right)
$$

Here $C_{i+1}\left(\epsilon_{u_{i+1}}\right)$ is the all possible arrangements of all the gravitini except $u_{j}$ and $u_{i}$ and similarly $C_{i}\left(\epsilon_{u_{i}}\right)$ is the all possible arrangements of all the gravitini except $u_{j}$ and $u_{i+1}$.

Now if we started with an ordering in which $u_{i+1}$ appeared before $u_{i}$ then we can repeat the same analysis. Equation (5.7) is same in both cases, but in (5.2) and in (5.3) will $i$ and $i+1$ will be interchanged (i.e. $i \longleftrightarrow i+1$ ). Hence instead of (5.6) we would get

$$
\left[A\left(\mathcal{S}_{u_{i+1}} \mathcal{S}_{u_{i}}+\mathcal{M}_{u_{i+1} u_{i}}\right) B\right] \Gamma_{N}\left(\left\{p_{i}\right\}\right)
$$

But now we can use (4.13) to see that (5.6) and (5.8) a essentially same. Hence the final answer is same irrespective of ordering of the soft factors.

\subsection{Gauge invariance}

We have proved the expression for multiple soft gravitini can be rearranged to any particular ordering. Using this, we can bring any gravitino to be the rightmost. So we will show the gauge invariance of the expression only when the rightmost gravitino is pure gauge.

The right-most gravitino can appear only in two ways

1. It can appear in $\mathcal{S}_{u}$. Since it is the right-most gravitino, it will directly hit the amplitude of the hard-particle and hence zero by (3.7).

2. Or it can appear in $\mathcal{M}_{u v}$. Again it will always appear as the 2nd index. But this vanishes because of (4.21a). 


\section{Two soft gravitini in the presence of central charge}

In case of extended supersymmetries, ${ }^{8}$ one can have central charges in the supersymmetry algebra. The supersymmetry algebra in (2.2) modifies to

$$
\left\{\mathcal{Q}_{\alpha}, \mathcal{Q}_{\beta}\right\}=-\frac{1}{2} \gamma_{\alpha \beta}^{\mu} P_{\mu}-\frac{1}{2} \mathcal{Z}_{\alpha \beta} \mathcal{U}
$$

$\mathcal{U}$ is (are) the generator(s) of $U(1)$ symmetry(-ies) generated by the central charge(s). As explained below equation (2.2), $\alpha, \beta$ are some (ir-)reducible spinor indices. In this language the existence of central charge is equivalent to the condition that there exists an element(s) $Z_{\alpha}{ }^{\beta}$ in the Clifford algebra such that, $\mathcal{Z}_{\alpha \beta}$ satisfies

$$
\mathcal{Z}_{\alpha \beta}=\mathcal{Z}_{\beta \alpha}
$$

In general, there can be higher form central charges. For example, in $D=11$, the supersymmetry algebra is of the form

$$
\left\{\mathcal{Q}_{\alpha}, \mathcal{Q}_{\beta}\right\}=-\frac{1}{2} \gamma_{\alpha \beta}^{\mu} P_{\mu}+\gamma_{\alpha \beta}^{\mu_{1} \mu_{2} \mu_{3}} A_{\mu_{1} \mu_{2} \mu_{3}}
$$

But for our purpose, we ignore any higher form central charges. This is because the higher form central charges can only minimally couple to extended objects (of appropriate dimensions), whereas we consider the scattering of only point-like states.

In this case the commutator of two soft operators in (4.12) is modified as follows

$$
\left[\mathcal{S}_{u}, \mathcal{S}_{v}\right]=-\frac{\kappa^{2}}{2} \sum_{i=1}^{N}\left[\epsilon_{\mu}^{(u) ; \alpha}\left(\not p_{i ; \alpha \beta}+e_{i} \mathcal{Z}_{\alpha \beta}\right) \epsilon_{\nu}^{(v) ; \beta} \frac{p_{i}^{\mu}}{p_{i} \cdot k_{u}} \frac{p_{i}^{\nu}}{p_{i} \cdot k_{v}}\right]
$$

In presence of the central term the computation in section 4 will be modified. In presence of particles charged under the central charge, the combined contribution from figure $3,4,5$ and 6 is not gauge invariant. We need a new interaction to make it gauge invariant. In fact, it is possible to extend the argument in [77] to argue that gauge invariance in presence of central charge implies the existence of massless photon which interacts at low energy.

Graviphoton and new interaction [89]. When we gauge the global supersymmetry with central charge to get supergravity, we get a $\mathrm{U}(1)^{N}$ gauge symmetry generated by spin 1 bosons (graviphoton) present in the graviton multiplet. These graviphotons couple to the gravitino and to any matter which carries the central charge. The coupling of the graviphoton to gravitino is completely fixed by supersymmetry and is related to that of graviton. The gravitino-gravitino-graviphoton three point function $\left(\widetilde{\mathcal{V}}^{\mu \nu ; \mu_{1}}\right)_{\alpha \beta}$ is given by

$$
-\mathbf{i} \kappa\left[k_{2}^{\mu_{1}}(\mathcal{Z})^{\alpha \beta} \eta^{\mu \nu}\right]-\frac{\mathbf{i} \kappa}{2}\left[\left[\left(k_{1}+k_{2}\right)_{c} \delta_{d}^{\mu_{1}}\right]\left(\mathcal{Z} \gamma^{c d}\right)^{\alpha \beta} \eta^{\mu \nu}\right]+\mathbf{i} \kappa\left[\left(k_{2}^{\mu} \eta^{\mu_{1} \nu}-k_{1}^{\nu} \eta^{\mu_{1} \mu}\right)(\mathcal{Z})^{\alpha \beta}\right]
$$

Whenever we have more than one soft gravitini, the vertex in (6.5) contributes. In particular, consider the case of two soft gravitini. We already evaluated it in section 4 . In presence of the central charge(s) we have a new contribution from the diagram 11

\footnotetext{
${ }^{8}$ We are thankful to Matteo Bertolini, Atish Dabholkar, Kyriakos Papadodimas, Cumrun Vafa for discussion on this point.
} 


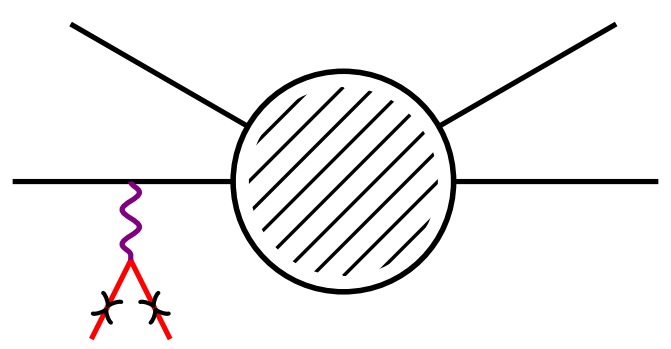

Figure 11. Feynman diagram for double soft gravitini - V.

Evaluation of figure 11. Now we would like to evaluate the figure 11. The evaluation of this diagram very similar to the evaluation of the figure 6 . It is given by

$$
\begin{aligned}
\Gamma_{N+2}^{(4)}\left(\left\{p_{i}\right\}, k_{1}, k_{2}\right)= & {\left[\epsilon_{\mu}^{(1) \alpha}\left(\widetilde{\mathcal{V}}^{\mu \nu ; \mu_{1}}\right)_{\alpha \beta} \epsilon_{\nu}^{(2) \beta}\right]\left[\frac{\mathbf{i} \eta_{\mu_{1} \mu_{2}}}{2 k_{1} \cdot k_{2}}\right] } \\
& \times\left[-2 \kappa e_{i} p_{i}^{\mu_{2}}\right]\left[\frac{1}{2 p_{i} \cdot\left(k_{1}+k_{2}\right)}\right] \Gamma_{N}\left(\left\{p_{i}\right\}\right)
\end{aligned}
$$

Here the first square bracket denotes gravitino-gravitino-graviphoton vertex, the second one is the graviphoton propagator and the third one is the matter- soft graviphoton matter vertex and the last one is the internal propagator. Now we substitute the explicit expression for $\left(\widetilde{\mathcal{V}}^{a b ; \mu}\right)_{\alpha \beta}$ from equation (6.5) and we obtain

$$
\begin{aligned}
& \Gamma_{N+2}^{(4)}(\left.\left(p_{i}\right\}, k_{1}, k_{2}\right) \\
&= \frac{(-\mathbf{i} \kappa)^{2}}{2} \sum_{i=1}^{N} e_{i} \epsilon_{\mu \alpha}^{(1)}(\mathcal{Z})^{\alpha \beta}\left[\eta^{\mu \nu} p_{i} \cdot k_{2}+\frac{1}{2} \eta^{\mu \nu}\left(k_{1}+k_{2}\right)_{d} p_{i e} \gamma^{d e}-\left(k_{2}^{\mu} p_{i}^{\nu}-k_{1}^{\nu} p_{i}^{\mu}\right)\right] \epsilon_{\nu \beta}^{(2)} \\
& \quad \times\left[\frac{1}{\left(p_{i} \cdot\left(k_{2}+k_{1}\right)\right)\left(k_{1} \cdot k_{2}\right)}\right] \Gamma_{N}\left(\left\{p_{i}\right\}\right)
\end{aligned}
$$

We simplify the above expression to get

$$
\begin{aligned}
& \Gamma_{N+2}^{(4)}(\left(\left\{p_{i}\right\}, k_{1}, k_{2}\right) \\
&= \frac{\kappa^{2}}{2} \sum_{i=1}^{N} e_{i} \epsilon_{\mu \alpha}^{(1)}(\mathcal{Z})^{\alpha \beta}\left[-\eta^{\mu \nu} p_{i} \cdot k_{2}+\frac{1}{2} \eta^{\mu \nu} p_{i} \cdot\left(k_{1}+k_{2}\right)+\left(k_{2}^{\mu} p_{i}^{\nu}-k_{1}^{\nu} p_{i}^{\mu}\right)\right] \epsilon_{\nu \beta}^{(2)} \\
& \quad \times\left[\frac{1}{\left(p_{i} \cdot\left(k_{2}+k_{1}\right)\right)\left(k_{1} \cdot k_{2}\right)}\right] \Gamma_{N}\left(\left\{p_{i}\right\}\right)
\end{aligned}
$$

In this case the definition of $\mathcal{C}_{u v}\left(p_{i}\right)$ in (4.8) will be modified as follows

$$
\widetilde{\mathcal{C}}_{u v}\left(p_{i}\right)=\mathcal{C}_{u v}\left(p_{i}\right)+\frac{\kappa^{2}}{2} e_{i} \epsilon_{\mu}^{(u)} \mathcal{Z} \epsilon_{\nu}^{(v)}\left[\frac{1}{2} \frac{\eta^{\mu \nu} p_{i} \cdot\left(k_{u}-k_{v}\right)}{k_{u} \cdot k_{v}}+\frac{\left(k_{v}^{\mu} p_{i}^{\nu}-k_{u}^{\nu} p_{i}^{\mu}\right)}{k_{u} \cdot k_{v}}\right]
$$

In equation (4.9) we show that $\mathcal{C}_{u v}$ is symmetric in its particle induces. The same property holds for $\widetilde{\mathcal{C}_{u v}}$

$$
\widetilde{\mathcal{C}}_{u v}\left(p_{i}\right)=\widetilde{\mathcal{C}}_{v u}\left(p_{i}\right)
$$


We add the contribution from (6.9) to (4.10) to get the final answer. It is given by

$$
\Gamma_{N+2}\left(\left\{p_{i}\right\}, k_{1}, k_{2}\right)=\left[\mathcal{S}_{1} \mathcal{S}_{2}+\widetilde{\mathcal{M}}_{12}\right] \Gamma_{N}\left(\left\{p_{i}\right\}\right)
$$

Here we have introduced $\widetilde{\mathcal{M}}_{u v}$. It is defined as

$$
\widetilde{\mathcal{M}}_{u v}=\mathcal{M}_{u v}+\frac{\kappa^{2}}{2} \sum_{i=1}^{N} e_{i} \frac{\epsilon_{\mu}^{(u)} \mathcal{Z} \epsilon_{\nu}^{(v)}}{p_{i} \cdot\left(k_{u}+k_{v}\right)}\left[\frac{p_{i}^{\mu} p_{i}^{\nu}}{p_{i} \cdot k_{v}}+\frac{1}{2} \frac{\eta^{\mu \nu} p_{i} \cdot\left(k_{u}-k_{v}\right)}{k_{u} \cdot k_{v}}+\frac{\left(k_{v}^{\mu} p_{i}^{\nu}-k_{u}^{\nu} p_{i}^{\mu}\right)}{k_{u} \cdot k_{v}}\right]
$$

Note that the relations in equations $(4.17 \mathrm{a}),(4.17 \mathrm{~b}),(4.17 \mathrm{c})$ remain the same if we replace $\mathcal{M}_{u v}$ with $\widetilde{\mathcal{M}}_{u v}$. In this particular case

$$
\mathcal{S}_{u} \mathcal{S}_{v}-\mathcal{S}_{v} \mathcal{S}_{u}=-\widetilde{\mathcal{M}}_{u v}+\widetilde{\mathcal{M}}_{v u}
$$

We check this explicitly. We have already the 1.h.s., i.e. $\mathcal{S}_{u} \mathcal{S}_{v}-\mathcal{S}_{v} \mathcal{S}_{u}$ in (6.4). Now we compute the r.h.s.

$$
\begin{aligned}
\widetilde{\mathcal{M}}_{v u}-\widetilde{\mathcal{M}}_{u v}= & \mathcal{M}_{v u}-\mathcal{M}_{u v} \\
& +\frac{\kappa^{2}}{2} \sum_{i=1}^{N} e_{i} \frac{\epsilon_{\mu}^{(v)} \mathcal{Z} \epsilon_{\nu}^{(u)}}{p_{i} \cdot\left(k_{u}+k_{v}\right)}\left[\frac{p_{i}^{\mu} p_{i}^{\nu}}{p_{i} \cdot k_{u}}+\frac{1}{2} \frac{\eta^{\mu \nu} p_{i} \cdot\left(k_{v}-k_{u}\right)}{k_{u} \cdot k_{v}}+\frac{\left(k_{u}^{\mu} p_{i}^{\nu}-k_{v}^{\nu} p_{i}^{\mu}\right)}{k_{u} \cdot k_{v}}\right] \\
& -\frac{\kappa^{2}}{2} \sum_{i=1}^{N} e_{i} \frac{\epsilon_{\mu}^{(u)} \mathcal{Z} \epsilon_{\nu}^{(v)}}{p_{i} \cdot\left(k_{u}+k_{v}\right)}\left[\frac{p_{i}^{\mu} p_{i}^{\nu}}{p_{i} \cdot k_{v}}+\frac{1}{2} \frac{\eta^{\mu \nu} p_{i} \cdot\left(k_{u}-k_{v}\right)}{k_{u} \cdot k_{v}}+\frac{\left(k_{v}^{\mu} p_{i}^{\nu}-k_{u}^{\nu} p_{i}^{\mu}\right)}{k_{u} \cdot k_{v}}\right] \\
= & -\frac{\kappa^{2}}{2} \sum_{i=1}^{N} \frac{\epsilon^{(u)} \cdot p_{i}}{p_{i} \cdot k_{u}} \frac{\epsilon^{(v)} \cdot p_{i}}{p_{i} \cdot k_{v}}\left(p_{i}+e_{i} \mathcal{Z}\right)
\end{aligned}
$$

Hence (6.13) is satisfied.

\subsection{Gauge invariance}

As explained in section 4.1, it is easier to prove gauge invariance if we put pure gauge polarization for the gravitino adjacent to $\Gamma_{N}$. So we consider pure gauge polarization for the second gravitino

$$
\epsilon_{2}^{\mu \alpha}=k_{2}^{\mu} \theta_{2}^{\alpha}
$$

For pure gauge

$$
\begin{aligned}
\widetilde{\mathcal{M}}_{u v}= & \frac{\kappa^{2}}{2} \sum_{i=1}^{N} \frac{1}{p_{i} \cdot\left(k_{u}+k_{v}\right)}\left[\epsilon_{\mu}^{(u)} \not p_{i} \theta^{(v)}+e_{i} \epsilon_{\mu}^{(u)} \mathcal{Z} \theta^{(v)}\right] \\
& \times\left[\frac{p_{i}^{\mu} p_{i} \cdot k_{v}}{p_{i} \cdot k_{v}}+\frac{1}{2} \frac{k_{v}^{\mu} p_{i} \cdot\left(k_{u}-k_{v}\right)}{k_{u} \cdot k_{v}}+\frac{\left(k_{v}^{\mu} k_{v} \cdot p_{i}-k_{v} \cdot k_{u} p_{i}^{\mu}\right)}{k_{u} \cdot k_{v}}\right] \\
= & \frac{\kappa^{2}}{2} \sum_{i=1}^{N} \frac{1}{p_{i} \cdot\left(k_{u}+k_{v}\right)}\left[\epsilon_{\mu}^{(u)} \not p_{i} \theta^{(v)}+e_{i} \epsilon_{\mu}^{(u)} \mathcal{Z} \theta^{(v)}\right]\left[\frac{1}{2} \frac{k_{v}^{\mu}}{k_{u} \cdot k_{v}}\right]=0
\end{aligned}
$$

where in the last step we have used momentum conservation and (central-)charge conservation

$$
\sum_{i=1}^{N} p_{i}=0 \quad, \quad \sum_{i=1}^{N} e_{i}=0
$$




\subsection{Presence of soft graviton}

Following $[1,2,68]$ it is easy to include soft graviton into this computation. The vertex for the leading soft graviton $\left(\zeta_{\mu \nu} P^{\mu} P^{\nu}\right)$ commutes with the vertex for soft gravitino and also commutes with the vertex for any other soft graviton. So, in the presence of $M_{1}$ soft gravitini and $M_{2}$ soft gravitons equation (5.1) is modified as follows

$$
\Gamma_{N+M_{1}+M_{2}}\left(\left\{p_{i}\right\},\left\{k_{r}\right\}\right)=\left[\prod_{j=1}^{M_{2}} \widetilde{\mathcal{S}}_{u_{j}}\right]\left[\prod_{i=1}^{M_{1}} \mathcal{S}_{u_{i}}+\sum_{A=1}^{\left\lfloor M_{1} / 2\right\rfloor} \prod_{i=1}^{A} \mathcal{M}_{u_{i} v_{i}} \prod_{j=1}^{M_{1}-2 A} \mathcal{S}_{r_{j}}\right] \Gamma_{N}\left(\left\{p_{i}\right\}\right)
$$

$\widetilde{\mathcal{S}}_{u}$ is the leading soft factor for graviton, given in equation (1.10).

We know that the leading and sub-leading soft factors for multiple gravitons are universal. In this paper, we derived the leading order expression for multiple soft gravitini and we found that it is also universal. These three soft theorems are inter-related by supersymmetry. One way to argue this is to observe that all these three soft theorems follow from covariantizing the action with respect to the soft field. In supergravity, the structure of the covariant derivative is uniquely fixed by supersymmetry.

\section{Infrared divergence and soft gravitino theorem}

Now we briefly discuss infrared divergences in supergravity theories. ${ }^{9}$ We are using 1PI effective action for our computation but this approach fails when 1PI vertices are IR divergent. The presence of massless particles in the loops can potentially give rise to these divergences, hence in the supergravity theories, graviton, gravi-photon and gravitino ${ }^{10}$ can contribute. There are no IR divergences in the $1 \mathrm{PI}$ effective action for $D \geq 5$. We will show below that the virtual gravitino does not give rise to any IR divergence in any dimensions. In $D=4,1 \mathrm{PI}$ vertices suffer from IR divergences only due to graviton and graviphoton. However, a more careful analysis shows that the leading soft gravitino factor is not altered by IR divergences.

First, we discuss the case of $D \geq 5$. Then we discuss the case of $D=4$ which needs more careful analysis. We show that IR divergence does not alter leading soft gravitino theorem.

\subsection{Infrared divergences in $D \geq 5$}

We want to check if the approach based on 1PI effective action remains valid in $D \geq 5$ even after taking the soft limit for the external gravitino.

Consider the Feynman diagram in figure 12(a), if the external momenta are finite, then by naive power-counting, we can see that the amplitude does not have IR divergence for $D \geq 4$. We have three powers of $\ell$ in the denominator, one from each of the propagators with momenta $p_{i}+\ell, p_{j}-\ell$ and $\ell$. The last propagator gives one power of $\ell$ because it

\footnotetext{
${ }^{9}$ We are thankful to Ashoke Sen for discussion on this section and correcting one mistake in an earlier version of the draft. We are thankful to the unknown referee for suggesting various improvements to this section.

${ }^{10}$ If there is a massless matter multiplet then in principle it can also contribute to infrared divergence.
} 


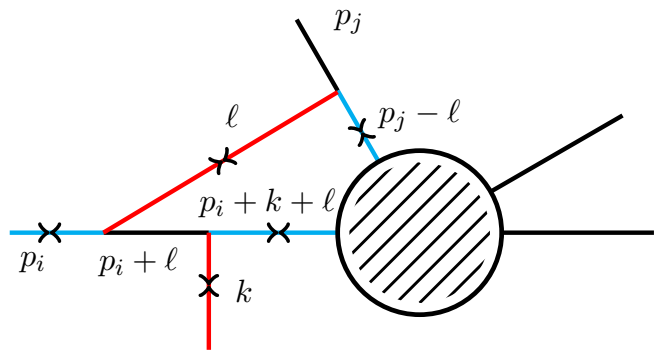

(a)

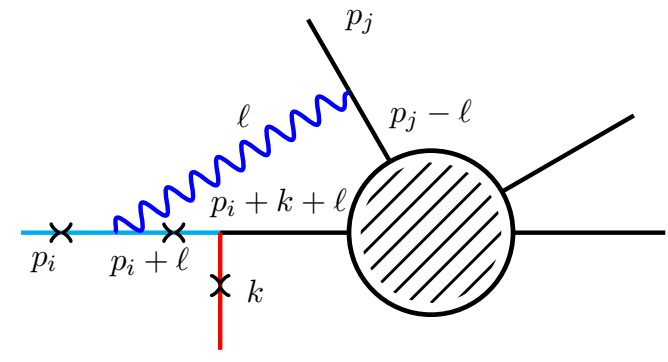

(b)

Figure 12. Infrared divergence in supergravity I.

is a fermionic particle. In $D$ dimensions, we have $D$ powers of $\ell$ in numerator due to the loop integral and hence the amplitude goes like $\ell^{D-3}$ for small loop momentum $\ell$. So the diagram is free of IR divergence in $D \geq 4$. Hence any virtual gravitino does not give rise to IR divergences. But when the momenta $k \rightarrow 0$, then the propagator carrying momentum $p_{i}+k+\ell$ gives another power of $\ell$ and makes the result logarithmically divergent in $D=4$ but there is no additional divergence in $D \geq 5$. So our results are still valid for $D \geq 5$.

Next we consider the Feynman diagram in figure 12(b). In this case, the internal massless particle is graviton (it can also be photon/graviphoton). From power-counting we see that there are four powers of $\ell$ in the denominator, one from each of the propagators with momenta $p_{i}+\ell, p_{j}-\ell$ and two powers of $\ell$ coming from the graviton propagator. Now in $k \rightarrow 0$ limit, the propagator carrying momentum $p_{i}+k+\ell$ gives another power of $\ell$ and the diagram is logarithmic divergent in $D=5$. But the leading order answer is $O\left(k^{-1}\right)$ and hence it still holds for $D \geq 5$.

\subsection{Infrared divergences in $D=4$}

In $D=4$, the 1PI effective action suffers from IR divergences due to the presence of graviton and photon in the loop (We already argued that there is no IR divergence in the 1PI vertex due to the presence of gravitino in the loop). So we cannot use it to compute the $S$-matrix. But one can use the tree level action to derive soft theorems order by order in perturbation theory. So in four dimensions, we use the tree level action instead of 1PI action in equation (2.3).

Now the question is whether loop corrections can alter the results of leading soft theorems. In case of soft graviton (and photon ${ }^{11}$ ), it has been shown that even though the amplitudes with and without soft particles suffer from IR divergences but at leading order, when one sum over diagrams, the divergence factorizes out and cancels from both sides $[7,25,40]$. In this section, we show that the same result holds for soft gravitino. We will show that the IR divergence due to graviton and graviphoton is same for amplitudes with and without soft gravitino.

\subsubsection{Single real soft gravitino in presence of virtual graviton}

First we consider the loop correction to the soft gravitino factor in $D=4$ in presence of a graviton running in the loop. We denote the contributions from these diagrams as

\footnotetext{
${ }^{11}$ With massive matter.
} 


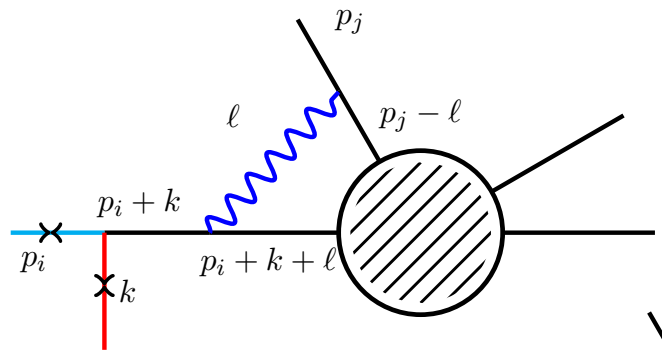

(a)

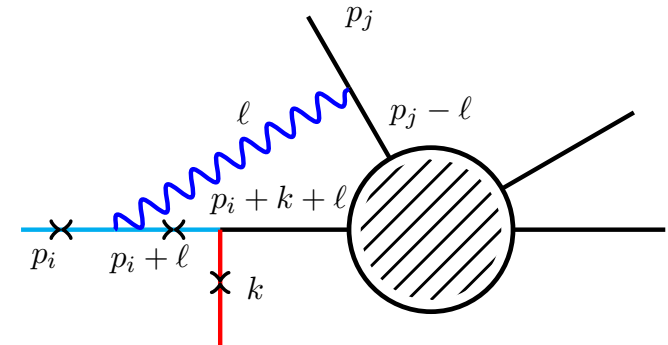

(b)

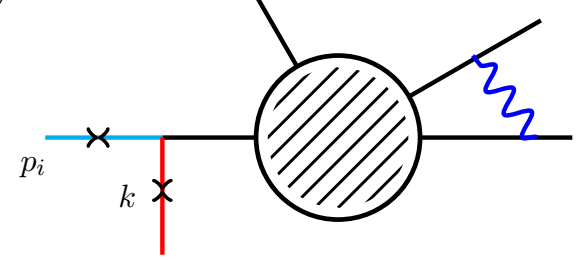

(c)

Figure 13. Infrared divergence in supergravity II.

$\Gamma_{N+1}^{(i ; j, k)}\left(k,\left\{p_{i}\right\}\right)$; here the superscripts $j$ and $k$ denote the legs to which the virtual graviton attaches and $i$ denote the one to which the soft gravitino attaches. The total contribution is given by

$$
\Gamma_{N+1}\left(k,\left\{p_{i}\right\}\right)=\sum_{i=1}^{N} \sum_{j=1}^{N} \sum_{k=1 ; k \neq j}^{N} \Gamma_{N+1}^{(i ; j, k)}\left(k,\left\{p_{i}\right\}\right)
$$

First we evaluate $\Gamma_{N+1}^{(i ; i, j)}\left(k,\left\{p_{i}\right\}\right)$. It is given by

$$
\Gamma_{N+1}^{(i ; i, j)}\left(k,\left\{p_{i}\right\}\right)=\left[\widetilde{A}_{1}\left(p_{i}, p_{j} ; k\right)+\widetilde{A}_{2}\left(p_{i}, p_{j} ; k\right)\right] \Gamma_{N}\left(\left\{p_{i}\right\}\right)
$$

$\widetilde{A}_{1}\left(p_{i}, p_{j} ; k\right)$ and $\widetilde{A}_{2}\left(p_{i}, p_{j} ; k\right)$ are contributions from diagram $(a)$ and $(b)$ respectively in figure 13. In small $k$ and small $\ell$ limit, these contributions are given by

$$
\begin{aligned}
& \widetilde{A}_{1}\left(p_{i}, p_{j} ; k\right)=\kappa^{3} \beta_{i j}\left(p_{i} \cdot \epsilon^{\alpha} \mathcal{Q}_{\alpha}\right) \int \frac{d^{4} \ell}{(2 \pi)^{4}} \frac{1}{\ell^{2}} \frac{1}{p_{i} \cdot \ell} \frac{1}{p_{j} \cdot \ell} \frac{1}{p_{i} \cdot(k+\ell)} \\
& \widetilde{A}_{2}\left(p_{i}, p_{j} ; k\right)=\kappa^{3} \beta_{i j}\left(p_{i} \cdot \epsilon^{\alpha} \mathcal{Q}_{\alpha}\right) \int \frac{d^{4} \ell}{(2 \pi)^{4}} \frac{1}{\ell^{2}} \frac{1}{p_{i} \cdot k} \frac{1}{p_{j} \cdot \ell} \frac{1}{p_{i} \cdot(k+\ell)}
\end{aligned}
$$

where $\beta_{i j}$ is given by

$$
\beta_{i j}=\left(\frac{\mathbf{i}}{2}\right)\left[2\left(p_{i} \cdot p_{j}\right)^{2}-p_{i}^{2} p_{j}^{2}\right]
$$

Adding the contributions from (7.3) and (7.4), we get

$$
\begin{aligned}
\Gamma_{N+1}^{(i ; i, j)}\left(k,\left\{p_{i}\right\}\right) & =\kappa^{2} \beta_{i j} \int \frac{d^{4} \ell}{(2 \pi)^{4}} \frac{1}{\ell^{2}} \frac{\kappa p_{i} \cdot \epsilon^{\alpha} \mathcal{Q}_{\alpha}}{p_{i} \cdot k} \frac{1}{p_{j} \cdot \ell} \frac{1}{p_{i} \cdot \ell} \Gamma_{N}\left(\left\{p_{i}\right\}+\mathcal{O}\left(k^{0}\right)\right. \\
& =A\left(p_{i}, p_{j}\right) \frac{\kappa p_{i} \cdot \epsilon^{\alpha} \mathcal{Q}_{\alpha}}{p_{i} \cdot k} \Gamma_{N}\left(\left\{p_{i}\right\}\right)+\mathcal{O}\left(k^{0}\right)
\end{aligned}
$$




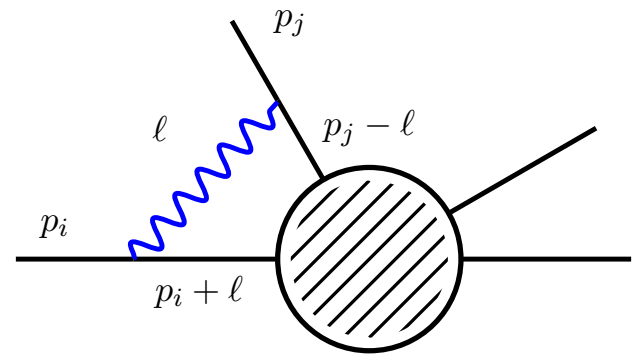

Figure 14. Infrared divergence in Supergravity III.

where $A\left(p_{i}, p_{j}\right)$ is the IR divergence that appears in diagram without soft gravitino which is depicted in figure 14. It is given by:

$$
A\left(p_{i}, p_{j}\right)=\kappa^{2} \beta_{i j} \int \frac{d^{4} \ell}{(2 \pi)^{4}} \frac{1}{\ell^{2}} \frac{1}{p_{i} \cdot \ell} \frac{1}{p_{j} \cdot \ell}
$$

The contribution from diagram $(c)$ in figure 13 is given by [7]

$$
\Gamma_{N+1}^{(i ; j \neq i, k \neq i)}\left(k,\left\{p_{i}\right\}\right)=A\left(p_{j}, p_{k}\right)\left[\frac{\kappa p_{i} \cdot \epsilon^{\alpha} \mathcal{Q}_{\alpha}}{p_{i} \cdot k}\right] \Gamma_{N}\left(\left\{p_{i}\right\}\right)
$$

Putting (7.6) and (7.8) in (7.1), we obtain

$$
\Gamma_{N+1}\left(k,\left\{p_{i}\right\}\right)=\left[\kappa \sum_{i=1}^{N} \frac{p_{i} \cdot \epsilon^{\alpha} \mathcal{Q}_{\alpha}}{p_{i} \cdot k}\right]\left[\sum_{j=1}^{N} \sum_{k=1, \neq j}^{N} A\left(p_{j}, p_{k}\right)\right] \Gamma_{N}\left(\left\{p_{i}\right\}\right)
$$

Here we found that the soft gravitino factor just factors out from the IR divergent integral.

Now we will compute the two loop contributions to IR divergence. We depicted the corresponding Feynman diagrams in figure 15. The contribution from these diagrams are given by

$$
\Gamma_{N+1}^{(i ; i, j)}\left(k,\left\{p_{i}\right\}\right)=\left[\sum_{a=1}^{6} \int d^{4} \ell_{1} d^{4} \ell_{2} I^{(a)}\right]\left[\kappa p_{i} \cdot \epsilon^{\alpha} \mathcal{Q}_{\alpha}\right] \Gamma_{N}\left(\left\{p_{i}\right\}\right)
$$

where $I^{(1,2,3)}$ are obtained as the integrands from the three diagrams shown below. The other three integrands are obtained from the non-planar diagram. The explicit expressions for these integrands are given by

$$
\begin{aligned}
I^{(1)} & =\kappa^{2} \frac{1}{p_{i} \cdot k} \frac{\beta_{i j}}{\ell_{2}^{2}} \frac{1}{p_{i} \cdot\left(k+\ell_{2}\right)} \frac{\beta_{i j}}{\ell_{1}^{2}} \frac{1}{p_{i} \cdot\left(k+\ell_{1}+\ell_{2}\right)} \frac{1}{p_{j} \cdot \ell_{2}} \frac{1}{p_{j} \cdot\left(\ell_{1}+\ell_{2}\right)} \\
I^{(2)} & =\kappa^{2} \frac{\beta_{i j}}{\ell_{2}^{2}} \frac{1}{p_{i} \cdot l_{2}} \frac{1}{p_{i} \cdot\left(k+\ell_{2}\right)} \frac{\beta_{i j}}{p_{i} \cdot\left(k+\ell_{1}+\ell_{2}\right)} \frac{1}{p_{j} \cdot \ell_{2}} \frac{1}{\ell_{1}^{2}} \frac{1}{p_{j} \cdot\left(\ell_{1}+\ell_{2}\right)} \\
I^{(3)} & =\kappa^{2} \frac{\beta_{i j}}{p_{i} \cdot \ell_{2}} \frac{\beta_{i j}}{p_{i} \cdot\left(\ell_{1}+\ell_{2}\right)} \frac{1}{p_{i} \cdot\left(k+\ell_{1}+\ell_{2}\right)} \frac{1}{p_{j} \cdot \ell_{2}} \frac{1}{\ell_{1}^{2}} \frac{1}{\ell_{2}^{2}} \frac{1}{p_{j} \cdot\left(\ell_{1}+\ell_{2}\right)}
\end{aligned}
$$

Adding the three contributions above and the contributions from the non-planar diagrams we obtain,

$$
I\left(p_{i}, p_{j} ; k\right)=\frac{1}{p_{i} \cdot k} \int d^{4} \ell_{1} d^{4} \ell_{2} I\left(p_{i}, p_{j} ; \ell_{1}, \ell_{2}\right)
$$




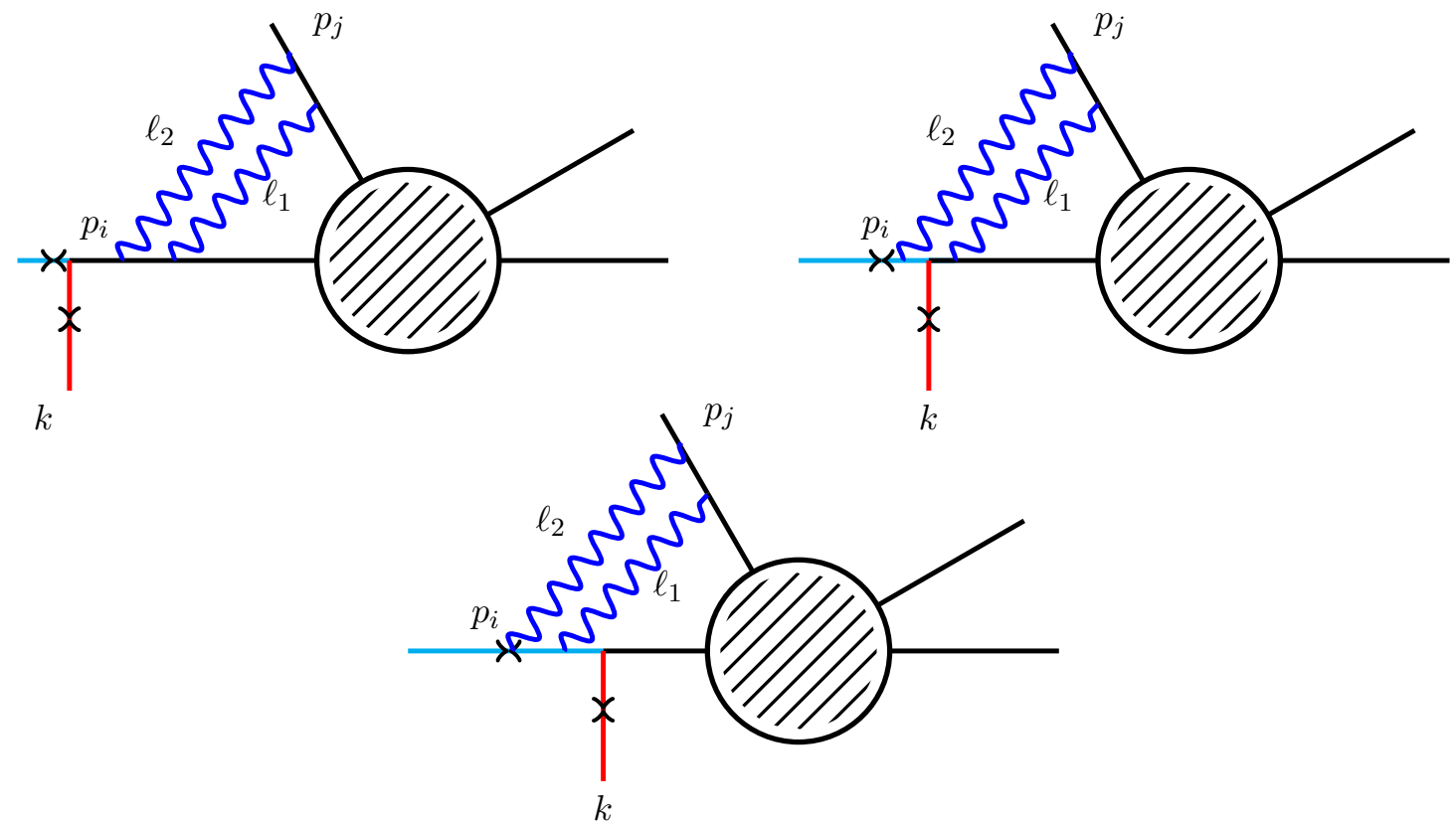

Figure 15. Infrared divergence in Supergravity IV.

where $I\left(p_{i}, p_{j} ; \ell_{1}, \ell_{2}\right)$ is given by

$$
I\left(p_{i}, p_{j} ; \ell_{1}, \ell_{2}\right)=\kappa^{2} \frac{1}{p_{i} \cdot\left(\ell_{1}+\ell_{2}\right)} \frac{1}{p_{i} \cdot \ell_{2}} \frac{1}{p_{j} \cdot \ell_{2}} \frac{\beta_{i j}}{\ell_{1}^{2}} \frac{\beta_{i j}}{\ell_{2}^{2}} \frac{1}{p_{j} \cdot \ell_{1}}
$$

which is the same two loop integrand we get when there is no soft gravitino. There are other two loop diagrams that we have not depicted here, for example, the diagrams in which two virtual gravitons attach to different legs etc. Adding contribution from all two loop diagrams we obtain

$$
\Gamma_{N+1}\left(k,\left\{p_{i}\right\}\right)=\frac{1}{2}\left[\sum_{j=1}^{N} \sum_{k=1 ; \neq j}^{N} A\left(p_{j}, p_{k}\right)\right]^{2}\left[\kappa \sum_{i=1}^{N} \frac{p_{i} \cdot \epsilon^{\alpha} \mathcal{Q}_{\alpha}}{p_{i} \cdot k}\right] \Gamma_{N}\left(\left\{p_{i}\right\}\right)
$$

Note that the soft factor appears just as a multiplicative factor with the infrared divergent piece. One can show that the contribution due to $N$ - virtual soft-gravitons and an external soft gravitino comes out to be

$$
\Gamma_{N+1}\left(k,\left\{p_{i}\right\}\right)=\left[\kappa \sum_{i=1}^{N} \frac{p_{i} \cdot \epsilon^{\alpha} \mathcal{Q}_{\alpha}}{p_{i} \cdot k}\right]\left[\sum_{N=0}^{\infty} \frac{1}{N !}\left[\sum_{j=1}^{N} \sum_{k=1 ; k \neq j}^{N} A\left(p_{j}, p_{k}\right)\right]^{N}\right] \Gamma_{N}\left(\left\{p_{i}\right\}\right)
$$

This implies the soft theorem is not affected by the IR divergence.

\subsubsection{Single real soft gravitino in presence of virtual graviphoton}

In the presence of graviphoton, there are new IR divergent diagrams due to graviphoton running in the loops. These diagrams can be obtained by replacing graviton with 
graviphoton in the figure 13 and in the figure 15. The computation is very similar to the one presented in subsection 7.1. The infrared divergence due to graviphoton is given by

$$
\left[\sum_{N=0}^{\infty} \frac{1}{N !}\left[B\left(p_{i}, p_{j}\right)\right]^{N}\right]
$$

where $B\left(p_{i}, p_{j}\right)$ is given by

$$
B\left(p_{i}, p_{j}\right)=\kappa^{2} e_{i} e_{j} \int \frac{d^{4} \ell}{(2 \pi)^{4}} \frac{1}{\ell^{2}} \frac{1}{p_{i} \cdot \ell} \frac{1}{p_{j} \cdot \ell} \frac{1}{p_{i} \cdot(k+\ell)}
$$

In presence of graviphoton, equation (7.15) will be replaced by the following equation

$$
\begin{aligned}
\Gamma_{N+1}\left(k,\left\{p_{i}\right\}\right)= & {\left[\kappa \sum_{i=1}^{N} \frac{p_{i} \cdot \epsilon^{\alpha} \mathcal{Q}_{\alpha}}{p_{i} \cdot k}\right] } \\
& \times\left[\sum_{N=0}^{\infty} \frac{1}{N !}\left[\sum_{j=1}^{N} \sum_{k=1 ; k \neq j}^{N}\left(A\left(p_{j}, p_{k}\right)+B\left(p_{j}, p_{k}\right)\right)\right]^{N}\right] \Gamma_{N}\left(\left\{p_{i}\right\}\right)
\end{aligned}
$$

Again we can see that the soft factor is not affected by the IR divergence.

\subsubsection{Massless matter}

Now we concentrate on the special case when some (or all) of the matter fields are massless. ${ }^{12}$ Weinberg in [7] showed that in the presence of massless matter the IR divergence due to virtual graviton cancels. However, there are irremovable IR divergences in QED with the massless matter.

In this case, the IR divergence comes from the presence of virtual graviton and virtual graviphoton. The ones due to virtual graviton cancel due to Weinberg's argument. However, in the presence of graviphoton, there might be some non-removable IR divergences. Graviphoton gauges the symmetries generated by the central charge. The central charge puts a lower bound on the mass of the particle (the BPS bound). The graviphoton only couples to matter with non-zero central charge and hence with non-zero mass. So there is no irremovable IR divergence in this case.

In presence of both the vector multiplet(s) and the massless matter multiplet(s) charged under the vector multiplet, there are irremovable IR divergences in $D=4$ due to the photon/gluon (of the vector multiplet) running in the loop. Since there is no vector multiplet in $\mathcal{N}=8$ supergravity, our analysis implies that there are no irremovable IR divergences in $\mathcal{N}=8$ supergravity (and in type II string theory).

\section{Conclusion}

In this paper, we have computed the multiple soft gravitini theorem at leading order in soft momenta for an arbitrary theory of supergravity. One natural question to ask is that what is the structure of the sub-leading soft gravitino theorem and how the structure of

\footnotetext{
${ }^{12}$ We are thankful to the unknown referee for pointing out this issue.
} 
the subleading soft gravitino theorem is related to that of sub-leading and sub-subleading soft graviton theorem. One can use this approach to compute soft photino theorem and correction to soft photino theorem in the presence of gravitino, photon, and graviton. Another interesting question is to derive the result for multiple soft gravitini from the analysis of asymptotic symmetries and from CFT living on $\mathscr{I}^{ \pm}$following [90-93]. Following [94] one could also try to verify this result from world-sheet methods. We leave these questions for future work.

\section{Acknowledgments}

We are thankful to Subramanya Hegde, Chandan Jana and Arnab P Saha for collaborating at the early stage of the project. We are thankful to Matteo Bertolini, Atish Dabholkar, Kyriakos Papadodimas, Giovanni Tambalo, Cumrun Vafa and especially Ashoke Sen for discussions at various stages. We are grateful to Atish Dabholkar, Nima Doroud, Subramanya Hegde, Chandan Jana, R Loganayagam, Usman Naseer and Mritunjay K Verma for reading the draft. We are grateful to Ashoke Sen going through the draft meticulously and for suggesting many improvements to an earlier version of this draft. Finally, we are grateful to the anonymous referee for many suggestions to improve this paper.

\section{A Notation and convention}

Our notation is as follows

$\begin{aligned} \text { Curved space indices } & \mu, \nu, \rho, \sigma \\ \text { Tangent space indices } & a, b \\ \text { SO }(d, 1) \text { spinor indices } & \alpha, \beta \\ \text { Soft-particle indices } & u, v \\ \text { Hard-particle indices } & i, j \\ \text { Number of Soft-particles } & M \\ \text { Number of Hard-particles } & N \\ \text { Polarization of the graviton } & \zeta_{\mu \nu} \\ \text { Polarization of the gravitino } & \epsilon_{\mu \alpha}\end{aligned}$

\section{A.1 Gamma matrix and spinor convention}

We use the following the gamma matrix convention

$$
\left\{\gamma^{a}, \gamma^{b}\right\}=-2 \eta^{a b}
$$

and we get

$$
\left[\gamma^{a}, \gamma^{b c}\right]=-2 \eta^{a b} \gamma^{c}+2 \eta^{a c} \gamma^{b}
$$

The basic spinors are defined as

$$
\psi_{\alpha}
$$


gamma matrix is

$$
\left(\gamma^{\mu}\right)_{\alpha}^{\beta}
$$

We raise and lower the indices as follows (NW-SE convention)

$$
\psi^{\alpha}=\mathcal{C}^{\alpha \beta} \psi_{\beta} \quad, \quad \psi_{\alpha}=\psi^{\beta} \mathcal{C}_{\beta \alpha}
$$

Here $\mathcal{C}^{\alpha \beta}$ satisfies

$$
\mathcal{C}^{\alpha \beta} \mathcal{C}_{\gamma \beta}=\delta_{\gamma}^{\alpha} \quad \mathcal{C}_{\beta \alpha} \mathcal{C}^{\beta \gamma}=\delta_{\alpha}^{\gamma}
$$

$\left(\gamma^{\mu}\right)_{\alpha \beta}$ is given by

$$
\gamma_{\alpha \beta}^{\mu}=\left(\gamma^{\mu}\right)_{\alpha}^{\gamma} \mathcal{C}_{\gamma \beta}
$$

\section{A.2 Majorana spinor}

For two Majorana spinors $\psi_{1}$ and $\psi_{2}$

$$
\left(\psi_{1}\right)^{\alpha}\left(\psi_{2}\right)_{\alpha}=\left(\psi_{2}\right)^{\alpha}\left(\psi_{1}\right)_{\alpha}
$$

\section{B Three soft gravitini}

In this appendix, we present the explicit computation for three soft gravitini. This computation is instructive to understand the soft factor for multiple gravitini, described in section 5 . In this section, we will write only $\Gamma_{N+3}$ instead of $\Gamma_{N+3}\left(\left\{p_{i}\right\}, k_{1}, k_{2}, k_{3}\right)$ to denote the amplitude with the soft gravitini and similarly we will write $\Gamma_{N}$ instead of $\Gamma_{N}\left(\left\{p_{i}\right\}\right)$ to denote the amplitudes involving only the hard-particles. For three soft gravitini, the different contributions are as follows:

- First consider Feynman diagrams where all three gravitini attach to separate external legs (figure 16). In this case the contribution will be just the multiplication of individual soft factors. So we get

$$
\Gamma_{N+3}^{(1)}=\kappa^{3} \sum_{i=1}^{N} \frac{p_{i}^{\mu} \epsilon_{\mu}^{(1) ; \alpha_{1}}}{p_{i} \cdot k_{1}} \mathcal{Q}_{\alpha_{1}} \sum_{j=1, j \neq i}^{N} \frac{p_{j}^{\mu} \epsilon_{\mu}^{(2) ; \alpha_{2}}}{p_{j} \cdot k_{2}} \mathcal{Q}_{\alpha_{2}} \sum_{k=1, k \neq i, j}^{N} \frac{p_{k}^{\mu} \epsilon_{\mu}^{(3) \alpha_{3}}}{p_{k} \cdot k_{3}} \mathcal{Q}_{\alpha_{3}} \Gamma_{N}\left(\left\{p_{i}\right\}\right)
$$

- Now we consider the case when two gravitini attach same leg and the third one on different leg as shown in figure 17. The contribution from such configurations is given by

$$
\Gamma_{N+3}^{u v \mid w ; 1}=\kappa^{3} \sum_{i=1}^{N} \frac{p_{i}^{\mu} \epsilon_{\mu}^{(u) \alpha_{u}}}{p_{i} \cdot k_{u}} \mathcal{Q}_{\alpha_{u}} \frac{p_{i}^{\mu} \epsilon_{\mu}^{(v) \alpha_{v}}}{p_{i} \cdot\left(k_{u}+k_{v}\right)} \mathcal{Q}_{\alpha_{v}} \sum_{j=1, j \neq i}^{N} \frac{p_{j}^{\mu} \epsilon_{\mu}^{(w) \alpha_{w}}}{p_{j} \cdot k_{w}} \mathcal{Q}_{\alpha_{w}} \Gamma_{N}\left(\left\{p_{i}\right\}\right)
$$

where $u, v, w$ can take values $1,2,3$. We can have different contributions depending on the order in which gravitini attach. 


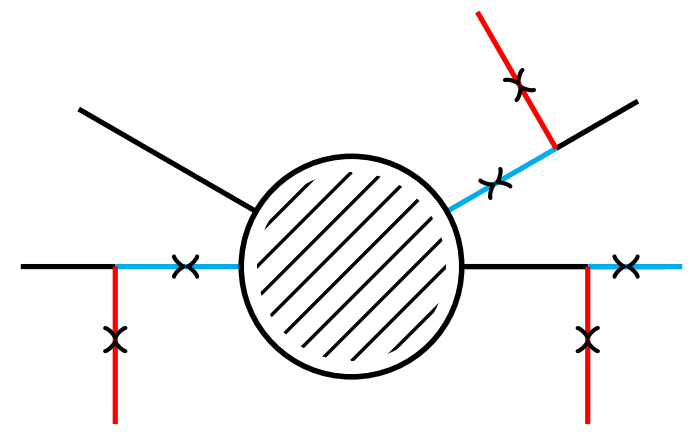

Figure 16. Feynman diagram for three soft gravitini - I.

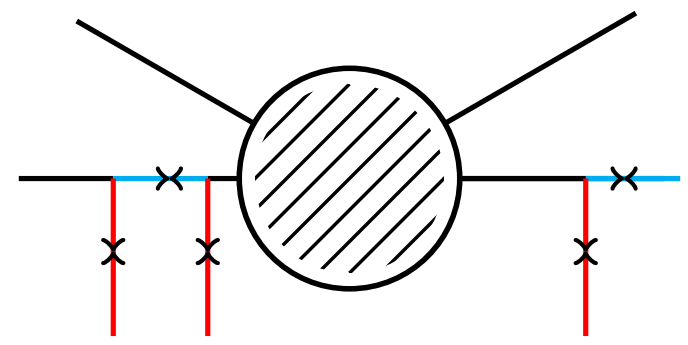

Figure 17. Feynman diagram for three soft gravitini - II.

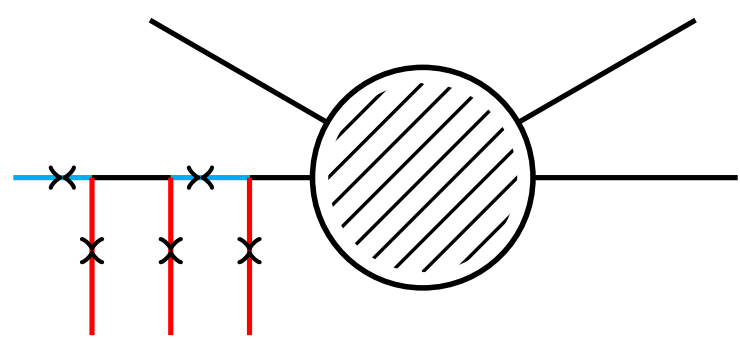

Figure 18. Feynman diagram for three soft gravitini - III.

- The third possibility consists of the diagrams when all gravitini are being attached to the same external leg (figure 18). The contribution is given by

$$
\Gamma_{N+3}^{u v w}=\kappa^{3} \sum_{i=1}^{N} \frac{\epsilon_{\mu}^{(u) \alpha_{u}} p_{i}^{\mu}}{p_{i} \cdot k_{u}} \frac{\epsilon_{\nu}^{(v) \alpha_{v}} p_{i}^{\nu}}{p_{i} \cdot\left(k_{u}+k_{v}\right)} \frac{\epsilon_{\rho}^{(w) \alpha_{w}} p_{i}^{\rho}}{p_{i} \cdot\left(k_{u}+k_{v}+k_{w}\right)} \mathcal{Q}_{\alpha_{u}} \mathcal{Q}_{\alpha_{v}} \mathcal{Q}_{\alpha_{w}} \Gamma_{N}\left(\left\{p_{i}\right\}\right)
$$

We have 6 diagrams which can be obtained by interchanging the external soft gravitini.

- Now we consider the diagrams in which any two soft gravitini combine to give a soft graviton and then the soft graviton attaches to the external leg; the left-over (lonely!) third one directly attaches to the external leg. This can also give rise to two scenarios, i.e. the internal soft graviton and the leftover lonely gravitino can attach to same hard particles or different hard particles. 


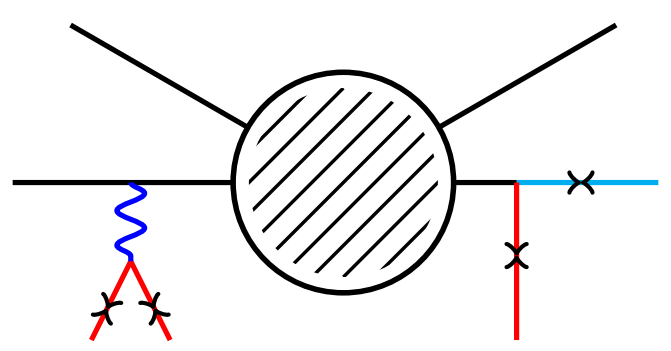

Figure 19. Feynman diagram for three soft gravitini - IV.

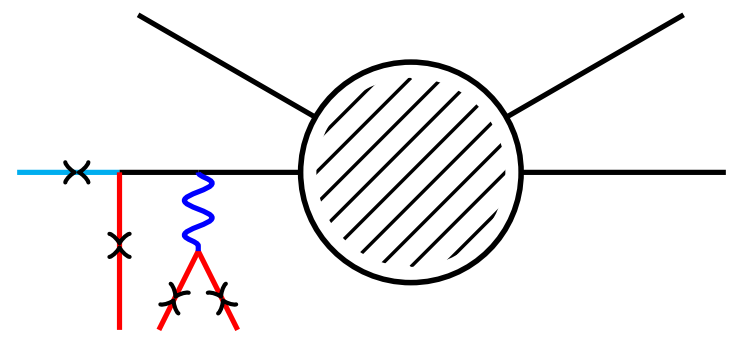

Figure 20. Feynman diagram for three soft gravitini - V.

In the case when they attach on separate legs as shown in figure 19, we just have the multiplication of two factors:

$$
\Gamma_{N+3}^{u v \mid w ; 2}=\kappa^{3} \sum_{i=1}^{N}\left[\frac{\mathcal{C}_{u v}\left(p_{i}\right)}{p_{i} \cdot\left(k_{u}+k_{v}\right)}\right] \sum_{j=1, j \neq i}^{N} \frac{\epsilon_{\mu}^{(w) \alpha_{w}} p_{j}^{\mu}}{p_{j} \cdot k_{w}} \mathcal{Q}_{\alpha_{w}} \Gamma_{N}\left(\left\{p_{i}\right\}\right)
$$

Since any two gravitini can combine to give the internal soft graviton (and the third one will attach to the separate leg), there are three possibilities.

Now we can have the case when both the internal soft graviton and the left-over soft gravitino attach to same external leg as shown in figure 20

$$
\Gamma_{N+3}^{u v \mid w ; 3}=\kappa^{3} \sum_{i=1}^{N}\left[\frac{\epsilon_{\mu}^{(w) \alpha_{w}} p_{j}^{\mu}}{p_{j} \cdot k_{w}} \mathcal{Q}_{\alpha_{w}} \frac{\mathcal{C}_{u v}\left(p_{i}\right)}{\left(p_{i} \cdot\left(k_{1}+k_{2}+k_{3}\right)\right)}\right] \Gamma_{N}\left(\left\{p_{i}\right\}\right)
$$

We will have another diagram in which the graviton attaches to the external leg first and then the gravitino attaches to the external leg i.e.

$$
\Gamma_{N+3}^{u v \mid w ; 4}=\kappa^{3} \sum_{i=1}^{N}\left[\frac{\mathcal{C}_{u v}\left(p_{i}\right)}{p_{i} \cdot\left(k_{u}+k_{v}\right)} \frac{\epsilon_{\mu}^{(w) \alpha_{w}} p_{j}^{\mu}}{p_{i} \cdot\left(k_{1}+k_{2}+k_{3}\right)} \mathcal{Q}_{\alpha_{w}}\right] \Gamma_{N}\left(\left\{p_{i}\right\}\right)
$$

Adding the contributions from (B.4), (B.5) and (B.6), we get

$$
\begin{aligned}
\Gamma_{N+3}^{u v \mid w ; 4} & =\Gamma_{N+3}^{u v \mid w ; 2}+\Gamma_{N+3}^{u v \mid w ; 3}+\Gamma_{N+3}^{u v \mid w ; 4} \\
& =\kappa^{2}\left[\left[\sum_{i=1}^{N} \frac{\mathcal{C}_{u v}\left(p_{i}\right)}{\left(p_{i} \cdot\left(k_{u}+k_{v}\right)\right)}\right] \mathcal{S}_{w}\right] \Gamma_{N}\left(\left\{p_{i}\right\}\right)
\end{aligned}
$$




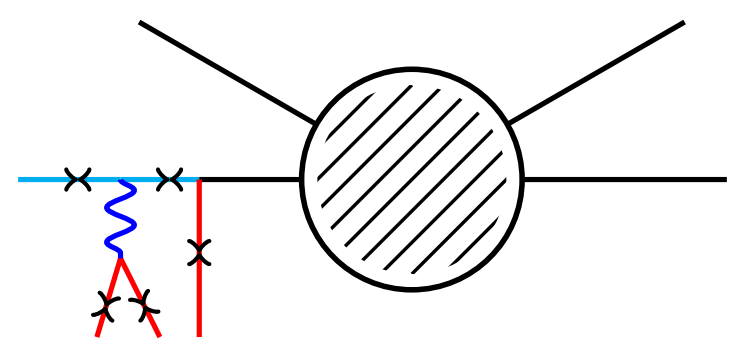

Figure 21. Feynman diagram for three soft gravitini — VI.

Now we write the contributions due to the six diagrams shown in figure 18 . We choose a particular ordering. We choose $\mathcal{Q}_{\gamma}$ to be the right-most. The $\Gamma_{N+3}^{123}$ remains the same

$$
\Gamma_{N+3}^{123}=\kappa^{3} \sum_{i=1}^{N} \frac{\epsilon_{\mu}^{(1) \alpha} p_{i}^{\mu}}{p_{i} \cdot k_{1}} \frac{\epsilon_{\nu}^{(2) \beta} p_{i}^{\nu}}{p_{i} \cdot\left(k_{1}+k_{2}\right)} \frac{\epsilon_{\rho}^{(3) \gamma} p_{i}^{\rho}}{p_{i} \cdot\left(k_{1}+k_{2}+k_{3}\right)} \mathcal{Q}_{\alpha} \mathcal{Q}_{\beta} \mathcal{Q}_{\gamma} \Gamma_{N}\left(\left\{p_{i}\right\}\right)
$$

Now we bring any other expression into this particular ordering by using (2.2). For example,

$$
\begin{aligned}
\Gamma_{N+3}^{132} & =\kappa^{3} \sum_{i=1}^{N} \frac{\epsilon_{\mu}^{(1) \alpha} p_{i}^{\mu}}{p_{i} \cdot k_{1}} \frac{\epsilon_{\nu}^{(3) \gamma} p_{i}^{\nu}}{p_{i} \cdot\left(k_{1}+k_{3}\right)} \frac{\epsilon_{\rho}^{(2) \beta} p_{i}^{\rho}}{p_{i} \cdot\left(k_{1}+k_{2}+k_{3}\right)} \mathcal{Q}_{\alpha} \mathcal{Q}_{\gamma} \mathcal{Q}_{\beta} \Gamma_{N}\left(\left\{p_{i}\right\}\right) \\
& =\kappa^{3} \sum_{i=1}^{N} \frac{\epsilon_{\mu}^{(1) \alpha} p_{i}^{\mu}}{p_{i} \cdot k_{1}} \frac{\epsilon_{\nu}^{(2) \beta} p_{i}^{\nu}}{p_{i} \cdot\left(k_{1}+k_{3}\right)} \frac{\epsilon_{\rho}^{(3) \gamma} p_{i}^{\rho}}{p_{i} \cdot\left(k_{1}+k_{2}+k_{3}\right)}\left[\mathcal{Q}_{\alpha} \mathcal{Q}_{\beta} \mathcal{Q}_{\gamma}+\frac{1}{2}\left(\not p_{i}\right)_{\beta \gamma} \mathcal{Q}_{\alpha}\right] \Gamma_{N}\left(\left\{p_{i}\right\}\right)
\end{aligned}
$$

Following the same philosophy, we obtain

$$
\begin{aligned}
\Gamma_{N+3}^{213}=\kappa^{3} \sum_{i=1}^{N} \frac{\epsilon_{\mu}^{(1) \alpha} p_{i}^{\mu}}{p_{i} \cdot k_{2}} \frac{\epsilon_{\nu}^{(2) \beta} p_{i}^{\nu}}{p_{i} \cdot\left(k_{1}+k_{2}\right)} \frac{\epsilon_{\rho}^{(3) \gamma} p_{i}^{\rho}}{p_{i} \cdot\left(k_{1}+k_{2}+k_{3}\right)} \\
\quad \times\left[\mathcal{Q}_{\alpha} \mathcal{Q}_{\beta} \mathcal{Q}_{\gamma}+\frac{1}{2}\left(\not p_{i}\right)_{\alpha \beta} \mathcal{Q}_{\gamma}\right] \Gamma_{N}\left(\left\{p_{i}\right\}\right) \\
\Gamma_{N+3}^{231}=\kappa^{3} \sum_{i=1}^{N} \frac{\epsilon_{\mu}^{(1) \alpha} p_{i}^{\mu}}{p_{i} \cdot k_{2}} \frac{\epsilon_{\nu}^{(2) \beta} p_{i}^{\nu}}{p_{i} \cdot\left(k_{3}+k_{2}\right)} \frac{\epsilon_{\rho}^{(3) \gamma} p_{i}^{\rho}}{p_{i} \cdot\left(k_{1}+k_{2}+k_{3}\right)} \\
\quad \times\left[\mathcal{Q}_{\alpha} \mathcal{Q}_{\beta} \mathcal{Q}_{\gamma}-\frac{1}{2}\left(\not p_{i}\right)_{\alpha \gamma} \mathcal{Q}_{\beta}+\frac{1}{2}\left(\not p_{i}\right)_{\alpha \beta} \mathcal{Q}_{\gamma}\right] \Gamma_{N}\left(\left\{p_{i}\right\}\right) \\
\Gamma_{N+3}^{321}=\kappa^{3} \sum_{i=1}^{N} \frac{\epsilon_{\mu}^{(1) \alpha} p_{i}^{\mu}}{p_{i} \cdot k_{3}} \frac{\epsilon_{\nu}^{(2) \beta} p_{i}^{\nu}}{p_{i} \cdot\left(k_{3}+k_{2}\right)} \frac{\epsilon_{\rho}^{(3) \gamma} p_{i}^{\rho}}{p_{i} \cdot\left(k_{1}+k_{2}+k_{3}\right)} \\
\quad \times\left[\mathcal{Q}_{\alpha} \mathcal{Q}_{\beta} \mathcal{Q}_{\gamma}-\frac{1}{2}\left(\not p_{i}\right)_{\alpha \gamma} \mathcal{Q}_{\beta}+\frac{1}{2}\left(\not p_{i}\right)_{\alpha \beta} \mathcal{Q}_{\gamma}+\frac{1}{2}\left(\not p_{i}\right)_{\beta \gamma} \mathcal{Q}_{\alpha}\right] \Gamma_{N}\left(\left\{p_{i}\right\}\right) \\
\Gamma_{N+3}^{312}=\kappa^{3} \sum_{i=1}^{N} \frac{\epsilon_{\mu}^{(1) \alpha} p_{i}^{\mu}}{p_{i} \cdot k_{3}} \frac{\epsilon_{\nu}^{(2) \beta} p_{i}^{\nu}}{p_{i} \cdot\left(k_{3}+k_{1}\right)} \frac{\epsilon_{\rho}^{(3) \gamma} p_{i}^{\rho}}{p_{i} \cdot\left(k_{1}+k_{2}+k_{3}\right)} \\
\quad \times\left[\mathcal{Q}_{\alpha} \mathcal{Q}_{\beta} \mathcal{Q}_{\gamma}-\frac{1}{2}\left(\not p_{i}\right)_{\alpha \gamma} \mathcal{Q}_{\beta}+\frac{1}{2}\left(\not p_{i}\right)_{\beta \gamma} \mathcal{Q}_{\alpha}\right] \Gamma_{N}\left(\left\{p_{i}\right\}\right)
\end{aligned}
$$


Adding all the contributions from equation (B.8), (B.9) (B.10a)-(B.10d) we get:

$$
\begin{aligned}
\Gamma_{N+3}^{e x}= & \kappa^{3} \sum_{i=1}^{N} \frac{\epsilon_{\mu}^{(1) \alpha} p_{i}^{\mu}}{p_{i} \cdot k_{1}} \frac{\epsilon_{\nu}^{(2) \beta} p_{i}^{\nu}}{p_{i} \cdot k_{2}} \frac{\epsilon_{\rho}^{(3) \gamma} p_{i}^{\rho}}{p_{i} \cdot k_{3}} \mathcal{Q}_{\alpha} \mathcal{Q}_{\beta} \mathcal{Q}_{\gamma} \Gamma_{N}\left(p_{i}\right) \\
& +\frac{1}{2} \kappa^{3} \sum_{i=1}^{N}\left[\frac{\epsilon_{\mu}^{(1) \alpha} p_{i}^{\mu}}{p_{i} \cdot k_{1}}\left(p_{i}\right)_{\beta \gamma} \mathcal{Q}_{\alpha} \frac{\epsilon_{\nu}^{(2) \beta} p_{i}^{\nu}}{p_{i} \cdot\left(k_{2}+k_{3}\right)}-\frac{\epsilon_{\mu}^{(1) \alpha} p_{i}^{\mu}}{p_{i} \cdot\left(k_{1}+k_{3}\right)} \frac{\epsilon_{\nu}^{(2) \beta} p_{i}^{\nu}}{p_{i} \cdot k_{2}}\left(p_{i}\right)_{\alpha \gamma} \mathcal{Q}_{\beta}\right. \\
& \left.-\frac{\epsilon_{\mu}^{(1) \alpha} p_{i}^{\mu}}{p_{i} \cdot\left(k_{3}+k_{2}\right)} \frac{\epsilon_{\nu}^{(2) \beta} p_{i}^{\nu}}{p_{i} \cdot k_{2}}\left(p_{i}\right)_{\alpha \beta} \mathcal{Q}_{\gamma}\right] \frac{\epsilon_{\rho}^{(3) \gamma} p_{i}^{\rho}}{p_{i} \cdot k_{3}} \Gamma_{N}\left(\left\{p_{i}\right\}\right)
\end{aligned}
$$

We add the contributions from (B.1), (B.2) and (B.7) to get the full answer. The full result can be written as

$$
\Gamma_{N+3}\left(\left\{p_{i}\right\},\left\{k_{u}\right\}\right)=\left[\mathcal{S}_{1} \mathcal{S}_{2} \mathcal{S}_{3}+\mathcal{M}_{12} \mathcal{S}_{3}+\mathcal{M}_{23} \mathcal{S}_{1}+\mathcal{M}_{13} \mathcal{S}_{2}\right] \Gamma_{N}\left(\left\{p_{i}\right\}\right)
$$

where $\mathcal{S}_{u}$ and $\mathcal{M}_{u v}$ is defined in (3.4) and (4.11) respectively. The above answer matches with the proposed answer (5.1) with $M=3$.

Rearrangement. We have written the answer for a particular ordering (1-2-3). In this case, we explicitly demonstrate the rearrangement. Let's say we want to write in the order 1-3-2. We apply the identity (4.13) for $u=2, v=3$

$$
\begin{aligned}
\Gamma_{N+3}\left(\left\{p_{i}\right\},\left\{k_{u}\right\}\right) & =\left[\mathcal{S}_{1}\left(\mathcal{S}_{3} \mathcal{S}_{2}-\mathcal{M}_{23}+\mathcal{M}_{32}\right)+\mathcal{M}_{12} \mathcal{S}_{3}+\mathcal{M}_{23} \mathcal{S}_{1}+\mathcal{M}_{13} \mathcal{S}_{2}\right] \Gamma_{N}\left(\left\{p_{i}\right\}\right) \\
& =\left[\mathcal{S}_{1} \mathcal{S}_{3} \mathcal{S}_{2}+\mathcal{M}_{12} \mathcal{S}_{3}+\mathcal{M}_{32} \mathcal{S}_{1}+\mathcal{M}_{13} \mathcal{S}_{2}\right] \Gamma_{N}\left(\left\{p_{i}\right\}\right)
\end{aligned}
$$

\section{B.1 Gauge invariance}

The gauge invariance of (B.12) is the easiest to show if we put pure gauge polarization for the last one, for example, 3rd gravitino in (B.12), 2nd gravitino in (B.13). Because the answer can always be rearranged to any particular ordering, we can always bring any particular gravitino to be the last entry. So, it's sufficient to show that the gauge invariance for the pure gauge polarization of the last one.

Let us consider (B.12) and pure gauge polarization for the 3rd gravitino. The first and the second term vanish as in equation (3.7) and the third \& the fourth term vanish because of (4.21a).

Symmetric form. Now we write the answer (B.13) in the form which is manifestly symmetric in all the gravitini

$$
\begin{aligned}
\Gamma_{N+3}\left(\left\{p_{i}\right\},\left\{k_{u}\right\}\right)=\left[\frac{1}{3 !} \mathcal{S}_{(1} \mathcal{S}_{2} \mathcal{S}_{3)}+\kappa^{2} \sum_{r \neq u \neq s} \sum_{i=1}^{N} \frac{1}{p_{i} \cdot\left(k_{r}+k_{u}\right)}\right. \\
\left.\times\left(\frac{\left(\epsilon^{(r)} \cdot p_{i}\right) p_{i}\left(\epsilon^{(u)} \cdot p_{i}\right) p_{i} \cdot\left(k_{r}-k_{u}\right)}{4\left(p_{i} \cdot k_{r}\right)\left(p_{i} \cdot k_{u}\right)}+\mathcal{C}_{u r}\left(p_{i}\right)\right) \mathcal{S}_{s}\right] \Gamma_{N}\left(\left\{p_{i}\right\}\right)
\end{aligned}
$$

Open Access. This article is distributed under the terms of the Creative Commons Attribution License (CC-BY 4.0), which permits any use, distribution and reproduction in any medium, provided the original author(s) and source are credited. 


\section{References}

[1] A. Sen, Soft theorems in superstring theory, JHEP 06 (2017) 113 [arXiv:1702.03934] [INSPIRE].

[2] A. Sen, Subleading soft graviton theorem for loop amplitudes, JHEP 11 (2017) 123 [arXiv: 1703.00024] [INSPIRE].

[3] M. Gell-Mann and M.L. Goldberger, Scattering of low-energy photons by particles of spin 1/2, Phys. Rev. 96 (1954) 1433 [inSPIRE].

[4] F.E. Low, Scattering of light of very low frequency by systems of spin 1/2, Phys. Rev. 96 (1954) 1428 [INSPIRE].

[5] F.E. Low, Bremsstrahlung of very low-energy quanta in elementary particle collisions, Phys. Rev. 110 (1958) 974 [INSPIRE].

[6] S. Weinberg, Photons and gravitons in s matrix theory: derivation of charge conservation and equality of gravitational and inertial mass, Phys. Rev. 135 (1964) B1049 [INSPIRE].

[7] S. Weinberg, Infrared photons and gravitons, Phys. Rev. 140 (1965) B516 [INSPIRE].

[8] D.J. Gross and R. Jackiw, Low-energy theorem for graviton scattering, Phys. Rev. 166 (1968) 1287 [INSPIRE].

[9] R. Jackiw, Low-energy theorems for massless bosons: photons and gravitons, Phys. Rev. 168 (1968) 1623 [INSPIRE].

[10] T.H. Burnett and N.M. Kroll, Extension of the low soft photon theorem, Phys. Rev. Lett. 20 (1968) 86 [INSPIRE].

[11] J.S. Bell and R. Van Royen, On the low-burnett-kroll theorem for soft-photon emission, Nuovo Cim. A 60 (1969) 62 [inSPIRE].

[12] S. Saito, Low-energy theorem for Compton scattering, Phys. Rev. 184 (1969) 1894 [INSPIRE].

[13] V. Del Duca, High-energy Bremsstrahlung theorems for soft photons, Nucl. Phys. B 345 (1990) 369 [INSPIRE].

[14] A. Strominger, Asymptotic symmetries of Yang-Mills theory, JHEP 07 (2014) 151 [arXiv:1308.0589] [INSPIRE].

[15] A. Strominger, On BMS invariance of gravitational scattering, JHEP 07 (2014) 152 [arXiv: 1312.2229] [INSPIRE].

[16] T. He, V. Lysov, P. Mitra and A. Strominger, BMS supertranslations and Weinberg's soft graviton theorem, JHEP 05 (2015) 151 [arXiv:1401.7026] [INSPIRE].

[17] A. Strominger and A. Zhiboedov, Gravitational memory, BMS supertranslations and soft theorems, JHEP 01 (2016) 086 [arXiv:1411.5745] [INSPIRE].

[18] D. Kapec, V. Lysov, S. Pasterski and A. Strominger, Higher-dimensional supertranslations and Weinberg's soft graviton theorem, arXiv:1502.07644 [INSPIRE].

[19] A. Strominger, Lectures on the infrared structure of gravity and gauge theory, arXiv: 1703.05448 [INSPIRE].

[20] D. Kapec, V. Lysov and A. Strominger, Asymptotic symmetries of massless QED in even dimensions, Adv. Theor. Math. Phys. 21 (2017) 1747 [arXiv:1412.2763] [INSPIRE]. 
[21] M. Pate, A.-M. Raclariu and A. Strominger, Gravitational memory in higher dimensions, JHEP 06 (2018) 138 [arXiv: 1712.01204] [INSPIRE].

[22] C.D. White, Factorization properties of soft graviton amplitudes, JHEP 05 (2011) 060 [arXiv:1103.2981] [INSPIRE].

[23] E. Casali, Soft sub-leading divergences in Yang-Mills amplitudes, JHEP 08 (2014) 077 [arXiv: 1404.5551] [INSPIRE].

[24] B.U.W. Schwab and A. Volovich, Subleading soft theorem in arbitrary dimensions from scattering equations, Phys. Rev. Lett. 113 (2014) 101601 [arXiv:1404.7749] [INSPIRE].

[25] Z. Bern, S. Davies and J. Nohle, On loop corrections to subleading soft behavior of gluons and gravitons, Phys. Rev. D 90 (2014) 085015 [arXiv: 1405.1015] [INSPIRE].

[26] S. He, Y.-t. Huang and C. Wen, Loop corrections to soft theorems in gauge theories and gravity, JHEP 12 (2014) 115 [arXiv: 1405.1410] [INSPIRE].

[27] A.J. Larkoski, Conformal invariance of the subleading soft theorem in gauge theory, Phys. Rev. D 90 (2014) 087701 [arXiv: 1405.2346] [INSPIRE].

[28] C.D. White, Diagrammatic insights into next-to-soft corrections, Phys. Lett. B 737 (2014) 216 [arXiv: 1406.7184] [INSPIRE].

[29] F. Cachazo and E.Y. Yuan, Are soft theorems renormalized?, arXiv:1405.3413 [INSPIRE].

[30] N. Afkhami-Jeddi, Soft graviton theorem in arbitrary dimensions, arXiv:1405.3533 [INSPIRE].

[31] B.U.W. Schwab, Subleading soft factor for string disk amplitudes, JHEP 08 (2014) 062 [arXiv: 1406.4172] [INSPIRE].

[32] P. Di Vecchia, R. Marotta and M. Mojaza, Soft theorem for the graviton, dilaton and the Kalb-Ramond field in the bosonic string, JHEP 05 (2015) 137 [arXiv:1502.05258] [INSPIRE].

[33] M. Bianchi, S. He, Y.-t. Huang and C. Wen, More on soft theorems: trees, loops and strings, Phys. Rev. D 92 (2015) 065022 [arXiv:1406.5155] [INSPIRE].

[34] J. Broedel, M. de Leeuw, J. Plefka and M. Rosso, Constraining subleading soft gluon and graviton theorems, Phys. Rev. D 90 (2014) 065024 [arXiv:1406.6574] [INSPIRE].

[35] A. Sabio Vera and M.A. Vazquez-Mozo, The double copy structure of soft gravitons, JHEP 03 (2015) 070 [arXiv: 1412.3699] [INSPIRE].

[36] M. Zlotnikov, Sub-sub-leading soft-graviton theorem in arbitrary dimension, JHEP 10 (2014) 148 [arXiv: 1407.5936] [INSPIRE].

[37] Y.-J. Du, B. Feng, C.-H. Fu and Y. Wang, Note on soft graviton theorem by KLT relation, JHEP 11 (2014) 090 [arXiv: 1408.4179] [INSPIRE].

[38] F. Cachazo, S. He and E.Y. Yuan, New double soft emission theorems, Phys. Rev. D 92 (2015) 065030 [arXiv: 1503.04816] [INSPIRE].

[39] C. Kalousios and F. Rojas, Next to subleading soft-graviton theorem in arbitrary dimensions, JHEP 01 (2015) 107 [arXiv: 1407.5982] [INSPIRE].

[40] Z. Bern, S. Davies, P. Di Vecchia and J. Nohle, Low-energy behavior of gluons and gravitons from gauge invariance, Phys. Rev. D 90 (2014) 084035 [arXiv:1406.6987] [InSPIRE].

[41] D. Bonocore et al., The method of regions and next-to-soft corrections in Drell-Yan production, Phys. Lett. B 742 (2015) 375 [arXiv:1410.6406] [INSPIRE]. 
[42] B.U.W. Schwab, A note on soft factors for closed string scattering, JHEP 03 (2015) 140 [arXiv: 1411.6661] [INSPIRE].

[43] T. Klose et al., Double-soft limits of gluons and gravitons, JHEP 07 (2015) 135 [arXiv: 1504.05558] [INSPIRE].

[44] A.E. Lipstein, Soft theorems from conformal field theory, JHEP 06 (2015) 166 [arXiv: 1504.01364] [INSPIRE].

[45] A. Volovich, C. Wen and M. Zlotnikov, Double soft theorems in gauge and string theories, JHEP 07 (2015) 095 [arXiv: 1504.05559] [INSPIRE].

[46] J. Rao and B. Feng, Note on identities inspired by new soft theorems, JHEP 04 (2016) 173 [arXiv: 1604.00650] [INSPIRE].

[47] P. Di Vecchia, R. Marotta and M. Mojaza, Double-soft behavior for scalars and gluons from string theory, JHEP 12 (2015) 150 [arXiv:1507.00938] [INSPIRE].

[48] M. Bianchi and A.L. Guerrieri, On the soft limit of open string disk amplitudes with massive states, JHEP 09 (2015) 164 [arXiv:1505.05854] [INSPIRE].

[49] A.L. Guerrieri, Soft behavior of string amplitudes with external massive states, Nuovo Cim. C 39 (2016) 221 [arXiv:1507.08829] [InSPIRE].

[50] Y.-t. Huang and C. Wen, Soft theorems from anomalous symmetries, JHEP 12 (2015) 143 [arXiv: 1509.07840] [inSPIRE].

[51] S.D. Alston, D.C. Dunbar and W.B. Perkins, n-point amplitudes with a single negative-helicity graviton, Phys. Rev. D 92 (2015) 065024 [arXiv:1507.08882] [INSPIRE].

[52] M. Bianchi and A.L. Guerrieri, On the soft limit of closed string amplitudes with massive states, Nucl. Phys. B 905 (2016) 188 [arXiv:1512.00803] [INSPIRE].

[53] P. Di Vecchia, R. Marotta and M. Mojaza, Soft theorems from string theory, Fortsch. Phys. 64 (2016) 389 [arXiv: 1511.04921] [INSPIRE].

[54] M. Bianchi and A.L. Guerrieri, On the soft limit of tree-level string amplitudes, in the proceedings of the $14^{\text {th }}$ Marcel Grossmann Meeting on Recent Developments in Theoretical and Experimental General Relativity, Astrophysics and Relativistic Field Theories (MG14), July 12-18, Rome, Italy (2015), arXiv: 1601.03457 [INSPIRE].

[55] P. Di Vecchia, R. Marotta and M. Mojaza, Subsubleading soft theorems of gravitons and dilatons in the bosonic string, JHEP 06 (2016) 054 [arXiv: 1604.03355] [INSPIRE].

[56] S. He, Z. Liu and J.-B. Wu, Scattering equations, twistor-string formulas and double-soft limits in four dimensions, JHEP 07 (2016) 060 [arXiv: 1604.02834] [INSPIRE].

[57] F. Cachazo, P. Cha and S. Mizera, Extensions of theories from soft limits, JHEP 06 (2016) 170 [arXiv: 1604.03893] [INSPIRE].

[58] A.P. Saha, Double soft theorem for perturbative gravity, JHEP 09 (2016) 165 [arXiv: 1607.02700] [INSPIRE].

[59] P. Di Vecchia, R. Marotta and M. Mojaza, Soft behavior of a closed massless state in superstring and universality in the soft behavior of the dilaton, JHEP 12 (2016) 020 [arXiv: 1610.03481] [INSPIRE].

[60] P. Di Vecchia, R. Marotta and M. Mojaza, Double-soft behavior of the dilaton of spontaneously broken conformal invariance, JHEP 09 (2017) 001 [arXiv:1705.06175] [INSPIRE]. 
[61] C. Cheung et al., A periodic table of effective field theories, JHEP 02 (2017) 020 [arXiv: 1611.03137] [INSPIRE].

[62] A. Luna, S. Melville, S.G. Naculich and C.D. White, Next-to-soft corrections to high energy scattering in QCD and gravity, JHEP 01 (2017) 052 [arXiv: 1611.02172] [INSPIRE].

[63] H. Elvang, C.R.T. Jones and S.G. Naculich, Soft photon and graviton theorems in effective field theory, Phys. Rev. Lett. 118 (2017) 231601 [arXiv:1611.07534] [INSPIRE].

[64] A.P. Saha, Double soft limit of the graviton amplitude from the Cachazo-He-Yuan formalism, Phys. Rev. D 96 (2017) 045002 [arXiv: 1702.02350] [INSPIRE].

[65] S. Chakrabarti et al., Subleading soft theorem for multiple soft gravitons, JHEP 12 (2017) 150 [arXiv: 1707.06803] [INSPIRE].

[66] Y. Hamada and G. Shiu, Infinite set of soft theorems in gauge-gravity theories as Ward-Takahashi identities, Phys. Rev. Lett. 120 (2018) 201601 [arXiv:1801.05528] [INSPIRE].

[67] Z.-Z. Li, H.-H. Lin and S.-Q. Zhang, Infinite soft theorems from gauge symmetry, Phys. Rev. D 98 (2018) 045004 [arXiv: 1802.03148] [INSPIRE].

[68] A. Laddha and A. Sen, Sub-subleading soft graviton theorem in generic theories of quantum gravity, JHEP 10 (2017) 065 [arXiv:1706.00759] [INSPIRE].

[69] F. Cachazo and A. Strominger, Evidence for a new soft graviton theorem, arXiv:1404.4091 [INSPIRE].

[70] S. Atul Bhatkar and B. Sahoo, Subleading soft theorem for arbitrary number of external soft photons and gravitons, JHEP 01 (2019) 153 [arXiv:1809.01675] [INSPIRE].

[71] F. Cachazo, S. He and E.Y. Yuan, Scattering of massless particles in arbitrary dimensions, Phys. Rev. Lett. 113 (2014) 171601 [arXiv:1307.2199] [InSPIRE].

[72] F. Cachazo, S. He and E.Y. Yuan, Scattering of massless particles: scalars, gluons and gravitons, JHEP 07 (2014) 033 [arXiv: 1309.0885] [INSPIRE].

[73] F. Cachazo, S. He and E.Y. Yuan, Scattering equations and matrices: from Einstein to Yang-Mills, DBI and NLSM, JHEP 07 (2015) 149 [arXiv: 1412.3479] [INSPIRE].

[74] F. Cachazo, S. He and E.Y. Yuan, Scattering equations and Kawai-Lewellen-Tye orthogonality, Phys. Rev. D 90 (2014) 065001 [arXiv:1306.6575] [INSPIRE].

[75] T.T. Dumitrescu, T. He, P. Mitra and A. Strominger, Infinite-dimensional fermionic symmetry in supersymmetric gauge theories, arXiv:1511.07429 [INSPIRE].

[76] M.T. Grisaru, H.N. Pendleton and P. van Nieuwenhuizen, Supergravity and the $S$ matrix, Phys. Rev. D 15 (1977) 996 [InSPIRE].

[77] M.T. Grisaru and H.N. Pendleton, Soft spin 3/2 fermions require gravity and supersymmetry, Phys. Lett. B 67 (1977) 323.

[78] M.T. Grisaru and H.N. Pendleton, Some properties of scattering amplitudes in supersymmetric theories, Nucl. Phys. B 124 (1977) 81 [INSPIRE].

[79] V. Lysov, Asymptotic fermionic symmetry from soft gravitino theorem, arXiv:1512.03015 [INSPIRE].

[80] S.G. Avery and B.U.W. Schwab, Residual local supersymmetry and the soft gravitino, Phys. Rev. Lett. 116 (2016) 171601 [arXiv:1512.02657] [INSPIRE]. 
[81] P.P. Kulish and L.D. Faddeev, Asymptotic conditions and infrared divergences in quantum electrodynamics, Theor. Math. Phys. 4 (1970) 745 [InSPIRE].

[82] D. Kapec, M. Perry, A.-M. Raclariu and A. Strominger, Infrared divergences in QED, revisited, Phys. Rev. D 96 (2017) 085002 [arXiv:1705.04311] [INSPIRE].

[83] B. Gabai and A. Sever, Large gauge symmetries and asymptotic states in QED, JHEP 12 (2016) 095 [arXiv: 1607.08599] [INSPIRE].

[84] S. Choi and R. Akhoury, BMS supertranslation symmetry implies Faddeev-Kulish amplitudes, JHEP 02 (2018) 171 [arXiv: 1712.04551] [INSPIRE].

[85] J. Ware, R. Saotome and R. Akhoury, Construction of an asymptotic S matrix for perturbative quantum gravity, JHEP 10 (2013) 159 [arXiv:1308.6285] [INSPIRE].

[86] S. Choi, U. Kol and R. Akhoury, Asymptotic dynamics in perturbative quantum gravity and BMS supertranslations, JHEP 01 (2018) 142 [arXiv:1708.05717] [INSPIRE].

[87] A. Sen, Background independence of closed superstring field theory, JHEP 02 (2018) 155 [arXiv: 1711.08468] [INSPIRE].

[88] S. Ferrara et al., Scalar multiplet coupled to supergravity, Phys. Rev. D 15 (1977) 1013 [INSPIRE].

[89] C.K. Zachos, $N=2$ supergravity theory with a gauged central charge, Phys. Lett. B 76 (1978) 329.

[90] S. Pasterski, S.-H. Shao and A. Strominger, Flat space amplitudes and conformal symmetry of the celestial sphere, Phys. Rev. D 96 (2017) 065026 [arXiv:1701.00049] [InSPIRE].

[91] S. Pasterski and S.-H. Shao, Conformal basis for flat space amplitudes, Phys. Rev. D 96 (2017) 065022 [arXiv: 1705.01027] [INSPIRE].

[92] C. Cheung, A. de la Fuente and R. Sundrum, $4 D$ scattering amplitudes and asymptotic symmetries from $2 D$ CFT, JHEP 01 (2017) 112 [arXiv:1609.00732] [INSPIRE].

[93] L. Donnay, A. Puhm and A. Strominger, Conformally soft photons and gravitons, JHEP 01 (2019) 184 [arXiv:1810.05219] [INSPIRE].

[94] S. Higuchi and H. Kawai, Universality of soft theorem from locality of soft vertex operators, Nucl. Phys. B 936 (2018) 400 [arXiv:1805.11079] [INSPIRE]. 